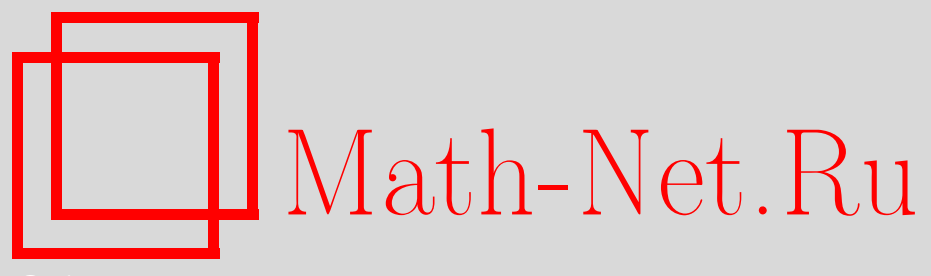

В. В. Горяйнов, Эволюционные семейства конформных отображений с неподвижными точками и уравнение Лёвнера-Куфарева, Матем. сб., 2015, том 206, номер 1, 39-68

DOI: https://doi.org/10.4213/sm8276

Использование Общероссийского математического портала Math-Net.Ru подразумевает, что вы прочитали и согласны с пользовательским соглашением http: //www . mathnet.ru/rus/agreement

Параметры загрузки:

IP : 54.89 .56 .158

26 апреля 2023 г., 17:04:36

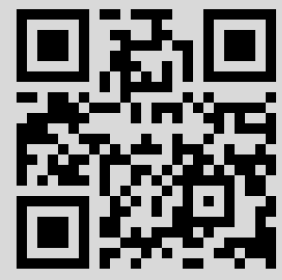




\title{
В. В. Горяйнов
}

\section{Эволюционные семейства конформных отображений с неподвижными точками и уравнение Лёвнера-Куфарева}

\begin{abstract}
Изучаются эволюционные семейства конформных отображений единичного круга в себя, имеющих внутреннюю и граничную неподвижные точки. Получены условия дифференцируемости эволюционных семейств и теорема существования и единственности для эволюционного уравнения. Установлена теорема сходимости, которая дает описание топологии локально равномерной сходимости эволюционных семейств в терминах инфинитезимальных производящих функций. Основной результат работы составляет теорема вложения, согласно которой всякое конформное отображение единичного круга в себя с двумя неподвижными точками можно вложить в дифференцируемое эволюционное семейство таких отображений. Этот результат позволяет расширить возможности параметрического метода теории однолистных функций. На этом пути решена задача о взаимном изменении производной во внутренней точке и угловой производной в граничной неподвижной точке для класса отображений единичного круга в себя. В частности, получена теорема вращения в этом классе отображений.
\end{abstract}

Библиография: 27 названий.

Ключевые слова: конформное отображение, неподвижная точка, эволюционное семейство, угловая производная, теорема вращения.

DOI: $10.4213 / \mathrm{sm} 8276$

\section{§ 1. Введение}

Трудности построения эффективного вариационного исчисления на классах однолистных функций во многом обусловлены существенной нелинейностью объекта исследования. Сумма двух однолистных функций не обязательно будет однолистной функцией. Поэтому даже задачи точных оценок линейных функционалов на классах однолистных функций (к таковым можно отнести гипотезу Бибербаха или проблему коэффициентов) оставались нерешенными долгое время. Это привело к возникновению и совершенствованию ряда специальных методов теории однолистных функций. Одним из наиболее плодотворных оказался параметрический метод Лёвнера (см. [1]), который привел к решению многих трудных задач теории однолистных функций. В частности, благодаря этому методу Г. М. Голузиным была установлена точная форма

Работа выполнена при поддержке Российского фонда фундаментальных исследований (грант № 12-01-00434-а). 
теоремы вращения (см. [2]) и Л. де Бранжем решена проблема коэффициентов (см. [3], [4]). Кроме того, Голузин в [5] показал, что этим методом могут быть единообразно получены решения многих внутренних задач теории однолистных функций. Термин "внутренние задачи" был введен Голузиным для выделения класса задач об оценках значений однолистной функции и ее производных в фиксированных точках единичного круга.

Принципиальным моментом метода Лёвнера, как отмечается в его классической работе [1], является то, что композиция однолистных отображений представляет собой снова однолистное отображение. Однако композицию двух отображений можно рассмотреть лишь в случае согласованности их областей определения и значений. Поэтому К. Лёвнер выделил класс $\mathfrak{L}$ голоморфных и однолистных отображений $f$ единичного круга $\mathbb{D}=\{z \in \mathbb{C}:|z|<1\}$ в себя, нормированных условиями $f(0)=0, f^{\prime}(0)>0$, и показал, что внутренние задачи теории однолистных функций можно свести к экстремальным задачам на классе $\mathfrak{L}$. Далее был поставлен вопрос: можно ли произвольное отображение $f$ из $\mathfrak{L}$ “составить" из инфинитезимальных преобразований? Под инфинитезимальными преобразованиями Лёвнер подразумевал функции вида $v(z)=-z p(z)$, где $p$ - голоморфная в $\mathbb{D}$ функция с неотрицательной вещественной частью и $p(0)>0$. В действительности это инфинитезимальные образующие однопараметрических полугрупп в $\mathfrak{L}$, т.е. непрерывных гомоморфизмов $t \mapsto f^{t}$, действующих из аддитивной полугруппы $\mathbb{R}_{+}=\{t \in \mathbb{R}: t \geqslant 0\}$ с обычной топологией вещественных чисел в топологическую полугруппу $\mathfrak{L}$ относительно операции композиции и топологии локально равномерной в $\mathbb{D}$ сходимости. Однопараметрическая полугруппа $t \mapsto f^{t}$ в $\mathfrak{L}$ определяет стационарный полупоток $t \mapsto f^{t}(z)$ в $\mathbb{D}$, который порождается автономным дифференциальным уравнением $d w / d t=-w p(w)$. Однако далеко не каждую функцию $f$ из $\mathfrak{L}$ можно вложить в однопараметрическую полугруппу. Поэтому Лёвнер рассмотрел неавтономное уравнение

$$
\frac{d w}{d t}=-w P(w, t)
$$

где $P(z, t)$ - непрерывная функция, определенная на $\mathbb{D} \times[0, T], T>0$, голоморфная по $z$ в $\mathbb{D}$, имеющая неотрицательную вещественную часть, и $P(0, t) \geqslant 0$. В работе [1] был изучен лишь частный случай уравнения (1), когда

$$
P(z, t)=\frac{1+\varkappa(t) z}{1-\varkappa(t) z},
$$

где $\varkappa(t)$ - непрерывная унимодулярная функция. Уравнение (1), в котором $P(z, t)$ является голоморфной с неотрицательной вещественной частью функцией по $z \in \mathbb{D}$, а по $t$ является измеримой, называют уравнением Лёвнера-Куфарева в связи с работой [6]. В работе [1] было показано, что функции $f(z)=$ $w_{T, 0}^{P}(z), T>0$, где $w=w_{t, 0}^{P}(z)$ - решение уравнения (1) с начальным условием $\left.w\right|_{t=0}=z$, образуют плотное в $\mathfrak{L}$ подмножество относительно топологии локально равномерной в $\mathbb{D}$ сходимости, если даже ограничиться случаем, когда $P(z, t)=(1+\varkappa(t) z) /(1-\varkappa(t) z)$. В случае общего вида $P(z, t)$, как следует из 
работ [7], [8], таким образом можно получить все функции $f$ из $\mathfrak{L}$. Отмеченное обстоятельство и составляет основу параметрического метода, который позволяет внутренние задачи теории однолистных функций сформулировать в виде задач оптимального управления, где роль управления играет функция $P(z, t)$.

Целью настоящей работы является развитие аналога параметрического метода для исследования внутренних задач теории однолистных функций с ограничениями на угловую производную в граничной точке. В связи с этим выделяется класс $\mathfrak{L}[0,1]$ голоморфных и однолистных отображений $f$ единичного круга $\mathbb{D}$ в себя, оставляющих неподвижным начало координат, т.е. $f(0)=0$, и граничную точку $z=1$ в смысле углового предела, а также имеющих в точке $z=1$ конечную угловую производную. В $\S 2$ приводится обсуждение понятий углового предела и угловой производной, устанавливаются некоторые результаты, связанные с этими понятиями, откуда, в частности, следует, что $\mathfrak{L}[0,1]$ является полугруппой относительно операции композиции. Отметим также, что $\mathfrak{L}[0,1]$ не является подполугруппой полугруппы $\mathfrak{L}$, поскольку для функций $f$ из $\mathfrak{L}[0,1]$ условие $f^{\prime}(0)>0$ может нарушаться.

В работе [9] были изучены однопараметрические полугруппы $t \mapsto f^{t}$ в $\mathfrak{L}[0,1]$ и установлен вид их инфинитезимальных образующих

$$
v(z)=\left.\frac{\partial}{\partial t} f^{t}(z)\right|_{t=0} .
$$

Для того чтобы голоморфная в $\mathbb{D}$ функция $v$ являлась инфинитезимальной образующей некоторой однопараметрической полугруппы $t \mapsto f^{t}$ в $\mathfrak{L}[0,1]$, необходимо и достаточно, чтобы она допускала представление в виде

$$
v(z)=-\alpha z(1-z) h(z)
$$

где $\alpha \geqslant 0$, а функция $h$ определяется формулой

$$
h(z)=\int_{\mathbb{T}} \frac{1-\varkappa}{1-\varkappa z} d \mu(\varkappa)
$$

с некоторой вероятностной (регулярной, борелевской с единичной общей массой) мерой $\mu$ на $\mathbb{T}=\{\varkappa \in \mathbb{C}:|\varkappa|=1\}$. Совокупность функций $h$, определяемых равенством (2), будем обозначать через $\mathscr{Q}$.

Расширение конструкции однопараметрической полугруппы с сохранением полугрупповых свойств естественно приводит к следующему понятию.

ОПредЕлЕниЕ. Двупараметрическое семейство $\left\{w_{t, s}: 0 \leqslant s \leqslant t \leqslant T\right\}$ полугруппы $\mathfrak{L}[0,1]$ будем называть эволюиионным семейством в $\mathfrak{L}[0,1]$ на промежутке $[0, T]$, если выполняются следующие условия:

(i) $w_{t, s}(z)=w_{t, \tau} \circ w_{\tau, s}(z)$ при $0 \leqslant s \leqslant \tau \leqslant t \leqslant T$;

(ii) $w_{t, s}(z) \rightarrow z$ локально равномерно в $\mathbb{D}$ при $(t-s) \rightarrow 0$.

В то время как дифференцируемость по параметру $t$ семейства функций $f^{t}$, $t>0$, однопараметрической полугруппы $t \mapsto f^{t}$ является внутренним свойством (см., например, [10]), дифференцируемость эволюционного семейства 
по $s$ и $t$ не следует непосредственно из определения. Прежде чем сформулировать результат об условиях дифференцируемости эволюционных семейств, заметим, что для любой функции $f$ из $\mathfrak{L}[0,1]$ угловая производная в точке $z=1$ удовлетворяет неравенству $f^{\prime}(1) \geqslant 1$ и знак равенства возможен лишь в случае $f(z) \equiv z$. Кроме того, из условия (i) в определении эволюционного семейства следует, что $\beta(t)=w_{t, 0}^{\prime}(1)$ является неубывающей на $[0, T]$ функцией и $\beta(t) \geqslant 1$.

Теорема 1. Пусть $\left\{w_{t, s}: 0 \leqslant s \leqslant t \leqslant T\right\}$ - эволюиионное семейство в $\mathfrak{L}[0,1]$ и $\beta(t)=w_{t, 0}^{\prime}(1)-$ абсолютно непрерьвная на $[0, T]$ функция. Тогда для любъх $z \in \mathbb{D}$ u $s \in[0, T)$ функиия $t \mapsto w_{t, s}(z)$ является абсолютно непрерывной на $[s, T]$ и для почти всех $t$ выполняется равенство

$$
\frac{\partial}{\partial t} w_{t, s}(z)=-\frac{\beta^{\prime}(t)}{\beta(t)} w_{t, s}(z)\left(1-w_{t, s}(z)\right) H\left(w_{t, s}(z), t\right),
$$

где $H(z, t)$ - функиия, определенная на $\mathbb{D} \times[0, T]$, голоморфная по $z$, измеримая nо $t$ и такая, что $H(\cdot, t) \in \mathscr{Q}$ для почти всех $t \in[0, T]$.

В условиях этой теоремы можно путем изменения временно́й шкалы получить нормированное эволюционное семейство, когда $\beta(t)=e^{t}, 0 \leqslant t \leqslant T$. При этом функция $t \mapsto w_{t, s}(z)$ будет решением (в смысле Каратеодори; см. [11]) дифференциального уравнения

$$
\frac{d w}{d t}=-w(1-w) H(w, t)
$$

с начальным условием $\left.w\right|_{t=s}=z$. Уравнение (3) будем называть эволюиионнъмм уравнением полугруппы $\mathfrak{L}[0,1]$, а функцию $H(z, t)$ - инфинитезимальной производящей функиией эволюционного семейства полугруппы $\mathfrak{L}[0,1]$. Уравнение (3) можно также считать аналогом уравнения Лёвнера-Куфарева. Прежде чем сформулировать теорему существования и единственности для этого уравнения, заметим, что для любой функции $h$ из $\mathscr{Q}$ существует угловой предел в точке $z=1$ и $0 \leqslant h(1) \leqslant 1$ (см. лемму 1 в $\S 2$ ).

Теорема 2. Пусть на $\mathbb{D} \times[0, T]$ определена комплекснозначная функиия $H(z, t)$, которая голоморфна по $z$, измерима по $t$ и удовлетворяет условию $H(\cdot, t) \in \mathscr{Q}$ для почти всех $t \in[0, T]$. Тогда для любых $z \in \mathbb{D} u s \in[0, T)$ существует единственное абсолютно непрерывное решение $w=w(t, z, s ; H)$, $s \leqslant t \leqslant T$, уравнения (3) с начальным условием $\left.w\right|_{t=s}=z$. Кроме того, при каждом $t \in[s, T]$ отображение $w_{t, s}^{H}: z \mapsto w(t, z, s ; H)$ является однолистным в $\mathbb{D}$, принадлежит $\mathfrak{L}[0,1]$ и его угловая производная в точке $z=1$ удовлетворяет соотношению

$$
\left(w_{t, s}^{H}\right)^{\prime}(1)=\exp \left\{\int_{s}^{t} H(1, \tau) d \tau\right\} .
$$

Следующий результат показывает, что каждое отображение $f$ из полугруппы $\mathfrak{L}[0,1]$ можно вложить в эволюционное семейство, которое порождается 
некоторой инфинитезимальной производящей функцией $H$, т.е. функцией, определенной на $\mathbb{D} \times[0, T]$ при некотором $T>0$ и удовлетворяющей условиям из теоремы 2 .

Tеорема 3. Пусть $f \in \mathfrak{L}[0,1] u f^{\prime}(1)=e^{T}, T>0$. Тогда найдется инбинитезимальная производящая функиия $H(z, t)$ полугруппы $\mathfrak{L}[0,1]$ на промежутке $[0, T]$ такая, что $w_{T, 0}^{H}(z)=f(z)$.

Одно из важных направлений геометрической теории функций комплексного переменного связано с получением точных оценок модуля и аргумента производной в различных классах однолистных функций. В связи с геометрическим смыслом производной конформного отображения эти результаты носят названия теорем искажения и вращения. Экстремальные функции в этих задачах дают представление о геометрии конформного отображения и имеют важное значение с точки зрения приложений, поскольку производная однолистной функции связана с комплексным потенциалом векторного поля. В последнее время в литературе значительное внимание уделяется получению оценок модуля производной однолистной (или многолистной) функции как во внутренней, так и в граничных точках единичного круга (см., например, [12]-[17]). В частности, во многих работах различными методами выводится неравенство в классе $\mathfrak{L}[0,1]$, которое связывает модули производных во внутренней и граничной точках. Теорема 3 позволяет решить более общую задачу о множестве значений системы функционалов на классе $\mathfrak{L}[0,1]$, которая содержит в себе как теорему искажения, так и теорему вращения. Таким образом, возникает аналог параметрического метода, который наряду с внутренними задачами (см. [5]) позволяет исследовать экстремальные задачи, включающие угловую производную однолистной функции в граничной точке.

Поскольку производная однолистной функции не обращается в нуль, то для любой $f$ из $\mathfrak{L}[0,1]$ можно выделить в $\mathbb{D}$ однозначную ветвь $\ln f^{\prime}(z)$. Замечая также, что угловая производная $f^{\prime}(1)$ принимает значение из промежутка $[1, \infty)$, под $\ln f^{\prime}(z)$ будем понимать ветвь, удовлетворяющую условию $\operatorname{Im}\left\{\ln f^{\prime}(r)\right\} \rightarrow 0$ при $r \nearrow 1$. С учетом выделенной ветви $\ln f^{\prime}(z)$ определим на классе $\mathfrak{L}[0,1]$ систему функционалов

$$
I(f)=\left\{\ln f^{\prime}(1), \ln f^{\prime}(0)\right\},
$$

а через $\mathscr{D}$ обозначим множество ее значений, т.е.

$$
\mathscr{D}=\left\{(\alpha, \zeta)=\left(\ln f^{\prime}(1), \ln f^{\prime}(0)\right): f \in \mathfrak{L}[0,1]\right\} .
$$

Очевидно, что $\mathscr{D} \subset \mathbb{R} \times \mathbb{C}$. Выделим также в $\mathfrak{L}[0,1]$ семейство функций следующим образом.

Для $\theta \in(-\pi / 2, \pi / 2)$ обозначим $\sigma=e^{i \theta}$ и определим функцию

$$
K_{\theta}(z)=\frac{z}{(1-z)^{1+\sigma^{2}}}
$$

где под степенной функцией понимается непрерывная ветвь, которая принимает значение 1 при $z=0$. Функция $K_{\theta}$ является голоморфной и однолистной 
в единичном круге $\mathbb{D}$, который она отображает на дополнение дуги логарифмической спирали $w(t)=-e^{t \sigma}, t \geqslant t^{*}(\theta)$, где

$$
t^{*}(\theta)=-2(\theta \sin \theta+\cos \theta \ln (2 \cos \theta)) .
$$

Далее, для $\tau>0$ определим функцию

$$
\phi_{\theta}^{\tau}(z)=K_{\theta}^{-1}\left(e^{-\tau \sigma} K_{\theta}(z)\right)
$$

которая принадлежит $\mathfrak{L}[0,1]$. Более детальное обсуждение свойств функций $K_{\theta}$ и $\phi_{\theta}^{\tau}$ приведено в $\S 6$.

Tеорема 4. Для того чтобъ точка $(\alpha, \zeta)$ из $\mathbb{R} \times \mathbb{C}$ принадлежала множеству $\mathscr{D}$ значений системы $I(f)$ на классе $\mathfrak{L}[0,1]$, необходимо и достаточно, чтобы выполнялись неравенства

$$
\alpha \geqslant 0, \quad|\zeta+\alpha| \leqslant \alpha .
$$

При этом точку $(\alpha, \zeta)$, для которой $|\zeta+\alpha|=\alpha, \alpha>0, \zeta \neq 0$, вносит единственная в $\mathfrak{L}[0,1]$ функиия $\phi_{\theta}^{\tau}$, где $\tau=|\zeta|$, , $\theta \in(-\pi / 2, \pi / 2)$ определяется из равенства

$$
e^{i 2 \theta}=-\frac{\zeta+\alpha}{\alpha} .
$$

Заметим, что если $f^{\prime}(1)=\beta>1$, то для $\zeta=\ln f^{\prime}(0)$ должно выполняться неравенство

$$
-2 \ln \beta \leqslant \operatorname{Re} \zeta=\ln \left|f^{\prime}(0)\right|,
$$

или, что эквивалентно, $\left|f^{\prime}(0)\right| \geqslant 1 / \beta^{2}$, а знак равенства достигается лишь для функции $\phi_{\theta}^{\tau}$ с параметрами $\theta=0$ и $\tau=2 \ln \beta$. Поскольку $K_{\theta}$ при $\theta=0$ представляет собой функцию Кёбе, то экстремаль $\phi_{0}^{\tau}$ является функцией Пика. Этот результат ранее был установлен различными методами в ряде работ (см., например, [13], [15]-[18]). Отметим также, что в монографии [19] изучалось взаимное изменение модулей функции и ее производной в фиксированных точках с ограничениями на угловую производную. Результат теоремы 4 содержит значительно больше информации. Так, оценка $\operatorname{Im} \zeta$ приводит к теореме вращения в классе $\mathfrak{L}[0,1]$, поскольку $\operatorname{Im}\left\{\ln f^{\prime}(0)\right\}=\arg f^{\prime}(0)$ выражает угол, на который поворачивается касательная при движении точки $w=f(x)$ вдоль образа вещественного радиуса $0 \leqslant x \leqslant 1$.

СлеДСТвиЕ. Пусть $f \in \mathfrak{L}[0,1]$ u $f(z) \not \equiv z$. Тогда имеет место неравенство

$$
\left|\arg f^{\prime}(0)\right| \leqslant \ln f^{\prime}(1)
$$

в котором знак равенства достигается только на функииях $\phi_{\theta}^{\tau}$, где $\tau=$ $\sqrt{2} \ln f^{\prime}(1) u \theta= \pm \pi / 4$.

Заметим также, что $K_{\theta}$ при $\theta= \pm \pi / 4$ отображает единичный круг на дополнение дуг логарифмических спиралей, у которых радиус-вектор образует с касательной угол, равный $\pm \pi / 4$. 


\section{§ 2. Окрестность тождественного преобразования и угловая производная}

В этом параграфе мы приведем некоторые обозначения и вспомогательные результаты, связанные с понятиями углового предела и угловой производной. Необходимые сведения по этим вопросам можно получить из монографий [20], [21].

В качестве угловой области в единичном круге $\mathbb{D}$ с вершиной в точке $z=1$ удобно рассмотреть окрестность радиуса $[0,1)$ в гиперболической метрике. Напомним, что гиперболическая метрика в $\mathbb{D}$ определяется элементом длины

$$
d s=\frac{2|d z|}{1-|z|^{2}}
$$

Геодезическими в этой метрике являются дуги окружностей, ортогональных единичной окружности $\mathbb{T}$. При этом гиперболическое расстояние между точками $z_{1}, z_{2}$ из $\mathbb{D}$ вычисляется по формуле

$$
d\left(z_{1}, z_{2}\right)=\ln \frac{\left|1-z_{1} \bar{z}_{2}\right|+\left|z_{1}-z_{2}\right|}{\left|1-z_{1} \bar{z}_{2}\right|-\left|z_{1}-z_{2}\right|}
$$

Множество $\Delta_{r}, 0 \leqslant r<1$, точек $z$ из $\mathbb{D}$, которые отстоят от радиуса $[0,1)$ в гиперболической метрике не более чем на $\ln ((1+r) /(1-r))$, представляет собой объединение

$$
\Delta_{r}=\left\{z \in \mathbb{D}: \frac{\left|1-z^{2}\right|}{1-|z|^{2}} \leqslant \frac{1+r^{2}}{1-r^{2}}, \operatorname{Re} z \geqslant 0\right\} \cup\{z \in \mathbb{D}:|z| \leqslant r\} .
$$

В частности, $\Delta_{0}=[0,1)$. Если $\varkappa \in \mathbb{T}$, то через $\Delta_{r}(\varkappa)$ будем обозначать поворот множества $\Delta_{r}$, т.е. $\Delta_{r}(\varkappa)=\left\{z \in \mathbb{D}: \bar{\varkappa} z \in \Delta_{r}\right\}$.

Пусть $f$ - голоморфная в $\mathbb{D}$ функция. Будем говорить, что в точке $\varkappa \in \mathbb{T}$ функция $f$ имеет угловой предел $\zeta$, если $f(z) \rightarrow \zeta$ при $z \rightarrow \varkappa, z \in \Delta_{r}(\varkappa)$, для всех $r \in[0,1)$. В этом случае будем писать

$$
\angle \lim _{z \rightarrow \varkappa} f(z)=\zeta
$$

Угловой предел $\zeta$ функции $f$ в точке $\varkappa$ естественно обозначать $f(\varkappa)$.

Лемма 1. Пусть функиия $h$ из класса $\mathscr{Q}$ имеет представление (2) с вероятностной мерой $\mu$ на $\mathbb{T}$. Тогда имеют место предельные соотношения

$$
\angle \lim _{z \rightarrow 1} h(z)=\mu(\mathbb{T} \backslash\{1\}), \quad \angle \lim _{z \rightarrow 1}(1-z) h^{\prime}(z)=0
$$

и для всех $z^{\prime}, z^{\prime \prime}$ из $\mathbb{D}$ выполняется неравенство

$$
\left|h\left(z^{\prime}\right)-h\left(z^{\prime \prime}\right)\right| \leqslant \frac{2\left|z^{\prime}-z^{\prime \prime}\right|}{\left(1-\left|z^{\prime}\right|\right)\left(1-\left|z^{\prime \prime}\right|\right)} .
$$


ДокАЗАТЕльство. Заметим вначале, что в действительности для любого $z \in \mathbb{D}$ имеет место равенство

$$
h(z)=\int_{\mathbb{T} \backslash\{1\}} \frac{1-\varkappa}{1-\varkappa z} d \mu(\varkappa) .
$$

Поэтому для доказательства первого предельного соотношения нам достаточно обосновать предельный переход под знаком интеграла при $z \rightarrow 1, z \in \Delta_{r}$. Пусть $0<r<1$ и $z \in \Delta_{r}$. Поскольку при отображении

$$
\varkappa \mapsto \frac{1-\varkappa}{1-\varkappa z}
$$

окружность $\mathbb{T}$ переходит в окружность с центром в точке $(1-\bar{z}) /\left(1-|z|^{2}\right)$, проходящую через начало координат, то для всех $\varkappa \in \mathbb{T}$ выполняется неравенство

$$
\left|\frac{1-\varkappa}{1-\varkappa z}\right| \leqslant \frac{2|1-z|}{1-|z|^{2}} .
$$

Из неравенств, определяющих угловую область $\Delta_{r}$, получаем также

$$
\frac{|1-z|}{1-|z|^{2}}=\frac{1}{|1+z|} \frac{\left|1-z^{2}\right|}{1-|z|^{2}} \leqslant \frac{1+r^{2}}{(1-r)\left(1-r^{2}\right)} .
$$

Таким образом, для всех $z \in \Delta_{r}$ и $\varkappa \in \mathbb{T}$ выполняется неравенство

$$
\left|\frac{1-\varkappa}{1-\varkappa z}\right| \leqslant \frac{2\left(1+r^{2}\right)}{(1+r)(1-r)^{2}},
$$

что означает выполнение условий теоремы Лебега о предельном переходе под знаком интеграла в определении функции $h$, и первое предельное соотношение доказано.

Далее, поскольку

$$
(1-z) h^{\prime}(z)=\int_{\mathbb{T}} \frac{\varkappa(1-\varkappa)(1-z)}{(1-\varkappa z)^{2}} d \mu(\varkappa)
$$

и при $z \in \Delta_{r}$ имеем

$$
\left|\frac{\varkappa(1-\varkappa)(1-z)}{(1-\varkappa z)^{2}}\right|=\left|\frac{1-\varkappa}{1-\varkappa z}\right| \frac{|1-z|}{|1-\varkappa z|} \leqslant 2\left|\frac{1-\varkappa}{1-\varkappa z}\right| \frac{|1-z|}{1-|z|^{2}} \leqslant \frac{4\left(1+r^{2}\right)^{2}}{(1+r)^{2}(1-r)^{4}},
$$

то снова можно осуществить предельный переход под знаком интеграла, что доказывает второе предельное соотношение.

Пусть теперь $z^{\prime}, z^{\prime \prime}$ - произвольные точки единичного круга. Тогда

$$
\left|h\left(z^{\prime}\right)-h\left(z^{\prime \prime}\right)\right|=\left|\int_{\mathbb{T}} \frac{\left(z^{\prime}-z^{\prime \prime}\right) \varkappa(1-\varkappa)}{\left(1-\varkappa z^{\prime}\right)\left(1-\varkappa z^{\prime \prime}\right)} d \mu(\varkappa)\right| \leqslant \frac{2\left|z^{\prime}-z^{\prime \prime}\right|}{\left(1-\left|z^{\prime}\right|\right)\left(1-\left|z^{\prime \prime}\right|\right)} .
$$

Лемма доказана. 
Обозначим через $\mathfrak{P}$ совокупность всех голоморфных отображений $f$ единичного круга $\mathbb{D}$ в себя. Для каждой $f$ из $\mathfrak{P}$ определим

$$
B(f)=\sup \left\{\frac{|1-f(z)|^{2}}{1-|f(z)|^{2}} \frac{1-|z|^{2}}{|1-z|^{2}}: z \in \mathbb{D}\right\} .
$$

Непосредственно из определения следует, что

$$
B(f) \geqslant \frac{|1-f(0)|^{2}}{1-|f(0)|^{2}} \geqslant \frac{1-|f(0)|}{1+|f(0)|} .
$$

Кроме того, $B(f)<\infty$ в том и только том случае, если

$$
\angle \lim _{z \rightarrow 1} f(z)=1, \quad \angle \lim _{z \rightarrow 1} \frac{f(z)-1}{z-1}=\angle \lim _{z \rightarrow 1} f^{\prime}(z)=B(f) .
$$

Поэтому в случае $B(f)<\infty$ эта величина называется угловой производной функции $f$ в точке $z=1$ и обозначается $f^{\prime}(1)$.

Лемма 2. Пусть $\left\{f_{n}\right\}$ - последовательность функиий из $\mathfrak{P}$ и $f_{n}(z) \rightarrow f(z)$ локально равномерно в $\mathbb{D}$ при $n \rightarrow \infty$. Если при этом $B\left(f_{n}\right)=\beta_{n} \leqslant M<\infty$ для всех $n=1,2, \ldots u|f(z)| \not \equiv 1$, mо $f \in \mathfrak{P} u$

$$
B(f) \leqslant \underline{\lim _{n \rightarrow \infty}} \beta_{n} .
$$

ДокАЗАТЕЛьство. В силу теоремы Жюлиа-Каратеодори для всех $z \in \mathbb{D}$ и $n=1,2, \ldots$ выполняется неравенство

$$
\frac{\left|1-f_{n}(z)\right|^{2}}{1-\left|f_{n}(z)\right|^{2}} \leqslant \beta_{n} \frac{|1-z|^{2}}{1-|z|^{2}} .
$$

Переходя в этом неравенстве к пределу по подпоследовательности $\left\{n_{k}\right\}$, на которой реализуется нижний предел последовательности $\left\{\beta_{n}\right\}$, получаем

$$
\frac{|1-f(z)|^{2}}{1-|f(z)|^{2}} \frac{1-|z|^{2}}{|1-z|^{2}} \leqslant \underline{\lim _{n \rightarrow \infty}} \beta_{n}
$$

что и доказывает лемму.

Обозначим через $\mathfrak{P}[0,1]$ совокупность функций $f$ из $\mathfrak{P}$, удовлетворяющих условиям $f(0)=0$ и $B(f)<\infty$, т.е. тех, которые оставляют неподвижными точки $z=0, z=1$ и имеют конечную угловую производную $f^{\prime}(1)$ в точке $z=1$. Из (5) следует, что для любой функции $f$ из $\mathfrak{P}[0,1]$ выполняется неравенство $f^{\prime}(1) \geqslant 1$. При этом равенство $f^{\prime}(1)=1$ возможно лишь в случае $f(z) \equiv z$. Действительно, если $f^{\prime}(1)=1$, то в силу теоремы Жюлиа-Каратеодори для всех $z \in \mathbb{D}$ выполняется неравенство

$$
\frac{|1-f(z)|^{2}}{1-|f(z)|^{2}} \leqslant \frac{|1-z|^{2}}{1-|z|^{2}}
$$

Отсюда при $z=x, 0<x<1$, получаем

$$
\frac{1-|f(x)|}{1+|f(x)|} \leqslant \frac{|1-f(x)|^{2}}{1-|f(x)|^{2}} \leqslant \frac{1-x}{1+x}
$$


и, следовательно, $|f(x)| \geqslant x$. С другой стороны, в силу леммы Шварца $|f(x)| \leqslant x$, и мы приходим к тождеству $f(z) \equiv z$.

Конечность угловой производной $f^{\prime}(1)$ приводит к соответствию орициклов при отображении единичного круга посредством функции $f$ из $\mathfrak{P}$. Под орициклом с параметром $\alpha>0$ будем понимать множество

$$
\mathbb{H}_{\alpha}=\left\{z \in \mathbb{D}: \frac{|1-z|^{2}}{1-|z|^{2}} \leqslant \alpha\right\}
$$

Если $f$ является голоморфным отображением единичного круга в себя, оставляет неподвижной точку $z=1$ и имеет в ней конечную угловую производную $f^{\prime}(1)=\beta$, то для любого $\alpha>0$ будет выполняться включение $f\left(\mathbb{H}_{\alpha}\right) \subset \mathbb{H}_{\alpha \beta}$. Следующий результат выражает соответствие угловых областей при отображении функциями класса $\mathfrak{P}[0,1]$.

Лемма 3. Пусть $f \in \mathfrak{P}[0,1] u f^{\prime}(1)=\beta>1$. Тогда для $r \in[0,1)$ выполняется включение $f\left(\Delta_{r}\right) \subset \Delta_{\rho}$, где

$$
\rho=\frac{r \sqrt{\beta+1}+\sqrt{\beta-1}}{\sqrt{\beta+1}+r \sqrt{\beta-1}} .
$$

ДокАЗАтельство. Рассмотрим вначале случай $r=0$, т.е. выясним степень искажения радиуса $[0,1)$. Для $x \in[0,1)$ в силу теоремы Жюлиа-Каратеодори выполняется неравенство

$$
\frac{|1-f(x)|^{2}}{1-|f(x)|^{2}} \leqslant \beta \frac{1-x}{1+x},
$$

из которого получаем следующее:

$$
\frac{\left|1-(f(x))^{2}\right|}{1-|f(x)|^{2}}=\frac{|1-f(x)|^{2}}{1-|f(x)|^{2}}\left|\frac{1+f(x)}{1-f(x)}\right| \leqslant \beta\left|\frac{(1-x)(1+f(x))}{(1+x)(1-f(x))}\right| .
$$

Заметим, что правая часть последнего неравенства является невозрастающей функцией по $x$ на промежутке $(0,1)$. Действительно, используя неравенство Пика, получаем

$$
\begin{aligned}
& \frac{d}{d x} \ln \left|\frac{(1-x)(1+f(x))}{(1+x)(1-f(x))}\right|=\frac{d}{d x} \operatorname{Re}\left\{\ln \frac{(1-x)(1+f(x))}{(1+x)(1-f(x))}\right\} \\
& =2 \operatorname{Re}\left\{\frac{f^{\prime}(x)}{1-(f(x))^{2}}-\frac{1}{1-x^{2}}\right\} \leqslant 2\left\{\frac{\left|f^{\prime}(x)\right|}{1-|f(x)|^{2}}-\frac{1}{1-x^{2}}\right\} \leqslant 0 .
\end{aligned}
$$

Таким образом, при $x \in[0,1)$ выполняется неравенство

$$
\frac{\left|1-(f(x))^{2}\right|}{1-|f(x)|^{2}} \leqslant \beta .
$$

Поскольку $\beta>1$, то $\theta=\sqrt{\beta-1} / \sqrt{\beta+1}$ принадлежит интервалу $(0,1)$ и $\beta=$ $\left(1+\theta^{2}\right) /\left(1-\theta^{2}\right)$. В этих терминах полученное выше неравенство означает, что гиперболическое расстояние от $f(x)$ до вещественного диаметра в $\mathbb{D}$ не 
превышает $\ln ((1+\theta) /(1-\theta))$. С другой стороны, $[0,1) \subset \mathbb{H}_{1}$ и, следовательно, $f([0,1)) \subset \mathbb{H}_{\beta}$. Заметим также, что

$$
\mathbb{H}_{\beta} \cap\left\{z \in \mathbb{D}: \frac{\left|1-z^{2}\right|}{1-|z|^{2}} \leqslant \frac{1+\theta^{2}}{1-\theta^{2}}=\beta\right\} \subset \Delta_{\theta} .
$$

Отсюда с использованием принципа гиперболической метрики и неравенства треугольника получаем включение $f\left(\Delta_{r}\right) \subset \Delta_{\rho}$, где $\rho$ определяется из равенства

$$
\ln \frac{1+\rho}{1-\rho}=\ln \frac{1+r}{1-r}+\ln \frac{1+\theta}{1-\theta} .
$$

Выражая отсюда $\rho$ через $\beta$ и $r$, приходим к утверждению леммы.

Из доказанной леммы 3 , в частности, следует, что если $f_{1}, f_{2} \in \mathfrak{P}[0,1]$, то и композиция $f=f_{1} \circ f_{2}$ также принадлежит $\mathfrak{P}[0,1]$ и $f^{\prime}(1)=f_{1}^{\prime}(1) f_{2}^{\prime}(1)$. Это означает, что $\mathfrak{P}[0,1]$ представляет собой полугруппу относительно операции композиции. При этом она является топологической полугруппой относительно топологии локально равномерной в $\mathbb{D}$ сходимости. Заметим также, что $\rho \rightarrow r$ при $\beta \rightarrow 1$ в формулировке леммы 3. Более того, в терминах угловой производной $f^{\prime}(1)$ описывается окрестность тождественного преобразования в полугруппе $\mathfrak{P}[0,1]$.

Лемма 4. Пусть $f \in \mathfrak{P}[0,1] u f^{\prime}(1)=\beta>1$. Тогда для всех $z \in \mathbb{D}$ выполняется неравенство

$$
|f(z)-z| \leqslant \frac{4|z|}{1-|z|}(\beta-1) .
$$

ДокАЗАТЕЛЬСтво. Поскольку $f(0)=0$, то функция $\varphi(z)=f(z) / z$ является голоморфной в $\mathbb{D}$ и $|\varphi(z)| \leqslant 1$ при $z \in \mathbb{D}$. Из условий леммы следует также, что $|\varphi(z)|<1$ при $z \in \mathbb{D}$, т.е. $\varphi \in \mathfrak{P}[0,1]$. При этом

$$
B(\varphi)=\angle \lim _{z \rightarrow 1} \frac{\varphi(z)-1}{z-1}=\angle \lim _{z \rightarrow 1}\left[\frac{1}{z}\left(\frac{f(z)-1}{z-1}-1\right)\right]=\beta-1 .
$$

В силу теоремы Жюлиа-Каратеодори для всех $z \in \mathbb{D}$ выполняется неравенство

$$
\frac{|1-\varphi(z)|^{2}}{1-|\varphi(z)|^{2}} \leqslant(\beta-1) \frac{|1-z|^{2}}{1-|z|^{2}}
$$

Поскольку

$$
\frac{|1-\varphi(z)|^{2}}{1-|\varphi(z)|^{2}} \geqslant \frac{(1-|\varphi(z)|)|1-\varphi(z)|}{1-|\varphi(z)|^{2}} \geqslant \frac{1}{2}|1-\varphi(z)|=\frac{1}{2}\left|\frac{f(z)-z}{z}\right|,
$$

то

$$
|f(z)-z| \leqslant 2(\beta-1)|z| \frac{|1-z|^{2}}{1-|z|^{2}} \leqslant 2(\beta-1)|z| \frac{|1-z|}{1-|z|} \leqslant \frac{4|z|}{1-|z|}(\beta-1),
$$

и лемма доказана. 


\section{§ 3. Эволюционные семейства}

Пусть $\left\{w_{t, s}: 0 \leqslant s \leqslant t \leqslant T\right\}-$ эволюционное семейство в полугруппе $\mathfrak{L}[0,1]$ на промежутке $[0, T]$. Рассмотрим однопараметрическое семейство функций $F(z, s)=w_{T, s}(z), 0 \leqslant s \leqslant T$. Из условия (i) в определении эволюционного семейства следует, что при $0 \leqslant s<t \leqslant T$ имеет место соотношение

$$
F(z, s)=w_{T, s}(z)=w_{T, t} \circ w_{t, s}(z)=F\left(w_{t, s}(z), t\right) .
$$

Это означает, что функции $w=F(z, s)$ конформно отображают единичный круг $\mathbb{D}$ на расширяющиеся области $D_{s}=F(\mathbb{D}, s), 0 \leqslant s \leqslant T$. При этом $D_{T}=\mathbb{D}$ и $F(z, T) \equiv z$. Условие (ii) из определения эволюционного семейства показывает, что семейство областей $D_{s}, 0 \leqslant s \leqslant T$, зависит от параметра $s$ непрерывно в смысле сходимости областей к ядру по Каратеодори.

Функцию $F(z, s)=w_{T, s}(z)$ будем называть производящей функиией эволюционного семейства $\left\{w_{t, s}: 0 \leqslant s \leqslant t \leqslant T\right\}$. Следует отметить, что перечисленные выше свойства $F(z, s)$ являются характеристическими для производящей функции и по ней однозначно восстанавливается эволюционное семейство посредством равенства

$$
w_{t, s}(z)=F^{-1}(F(z, s), t),
$$

$0 \leqslant s \leqslant t \leqslant T$. Вопрос дифференцируемости производящей функции $F(z, s)$ по параметру $s$ был решен в работе [22] в терминах угловой производной $F^{\prime}(1, s)$, где $F^{\prime}=\partial F / \partial z$. В частности, был установлен следующий результат.

Tеорема 5. Пусть $\left\{w_{t, s}: 0 \leqslant s \leqslant t \leqslant T\right\}-$ эволюиионное семейство полугруппы $\mathfrak{L}[0,1]$ на промежутке $[0, T]$ u $F(z, s)=w_{T, s}(z), 0 \leqslant s \leqslant T,-e е$ производящая функция. Тогда если $\gamma(s)=F^{\prime}(1, s)$ является абсолютно непрерывной на $[0, T]$ функиией, то для каждого $z \in \mathbb{D}$ функиия $s \mapsto F(z, s)$ также будет абсолютно непрерывной на $[0, T]$ и для почти всех s выполняется равенство

$$
\frac{\partial}{\partial s} F(z, s)=-\frac{\gamma^{\prime}(s)}{\gamma(s)} z(1-z) H(z, s) F^{\prime}(z, s),
$$

где $H(z, s)$ - функиия, определенная на $\mathbb{D} \times[0, T]$, голоморфная по $z$, измеримая nо s и такая, что $H(\cdot, s) \in \mathscr{Q}$ для почти всех $s \in[0, T]$.

Из этой теоремы легко выводится результат о дифференцируемости эволюционных семейств.

ДокаЗАтельСтво теоремы 1. Допустим, что $\left\{w_{t, s}: 0 \leqslant s \leqslant t \leqslant T\right\}-$ эволюционное семейство в полугруппе $\mathfrak{L}[0,1]$ и $\beta(t)=w_{t, 0}^{\prime}(1)$ является абсолютно непрерывной на $[0, T]$ функцией. Пусть также $F(z, s)=w_{T, s}, 0 \leqslant s \leqslant T$, ее производящая функция и $\gamma(s)=F^{\prime}(1, s)$. Поскольку для $0 \leqslant s<t \leqslant T$ выполняется соотношение

$$
w_{t, 0}(z)=w_{t, s} \circ w_{s, 0}(z)
$$

то $\beta(t)=\beta(s) w_{t, s}^{\prime}(1)$, откуда следует, что $\beta(t)$ является неубывающей на $[0, T]$ функцией и $w_{t, s}^{\prime}(1)=\beta(t) / \beta(s)$. Отсюда сразу же следуют равенство $\gamma(s)=$ 
$\beta(T) / \beta(s)$ и свойство абсолютной непрерывности функции $\gamma(s)$ на $[0, T]$. Таким образом, выполнены условия теоремы 5 .

Дифференцируя по $t$ соотношение

$$
F\left(w_{t, s}(z), t\right)=F(z, s)
$$

с учетом равенства (6), получаем

$$
F^{\prime}\left(w_{t, s}(z), t\right) \frac{\partial}{\partial t} w_{t, s}(z)-\frac{\gamma^{\prime}(t)}{\gamma(t)} w_{t, s}(z)\left(1-w_{t, s}(z)\right) H\left(w_{t, s}(z), t\right) F^{\prime}\left(w_{t, s}(z), t\right)=0 .
$$

Поскольку $F^{\prime}(z, t) \neq 0$ при $z \in \mathbb{D}$, то полученное равенство можно переписать в виде

$$
\frac{\partial}{\partial t} w_{t, s}(z)=\frac{\gamma^{\prime}(t)}{\gamma(t)} w_{t, s}(z)\left(1-w_{t, s}(z)\right) H\left(w_{t, s}(z), t\right) .
$$

Замечая также, что $\gamma^{\prime}(t) / \gamma(t)=-\beta^{\prime}(t) / \beta(t)$, приходим к доказываемому равенству. Теорема доказана.

Производящая функция имеет более простую структуру, чем эволюционное семейство. Поэтому конструирование эволюционного семейства проще осуществить через построение соответствующей производящей функции. Мы воспользуемся этим при решении задачи вложения отображения в эволюционное семейство в $\S 6$.

В заключение этого параграфа заметим, что в отличие от однопараметрической полугруппы, где допускается лишь линейная замена $t \mapsto \alpha t$ времени, эволюционное семейство допускает значительно более широкие возможности выбора временно́й шкалы. Пусть $\left\{w_{t, s}: 0 \leqslant s \leqslant t \leqslant T\right\}$ - эволюционное семейство полугруппы $\mathfrak{L}[0,1]$ и $\beta(t)=w_{t, 0}^{\prime}(1)$ - непрерывная на $[0, T]$ функция. Как отмечалось выше, $\beta(t)$ является неубывающей функцией и $\beta(0)=1$. Если для некоторых $t_{1}, t_{2}, 0 \leqslant t_{1}<t_{2} \leqslant T$, выполняется равенство $\beta\left(t_{1}\right)=\beta\left(t_{2}\right)$, то будем иметь $w_{t, s}^{\prime}(1)=1$ и $w_{t, s} \equiv z$ при $t_{1} \leqslant s \leqslant t \leqslant t_{2}$. Поэтому за счет сужения промежутка $[0, T]$ (удалением интервалов постоянства функции $\beta(t)$ ) можно добиться того, чтобы $\beta(t)$ была строго монотонно возрастающей функцией.

Далее, если $F(z, t)$ - производящая функция эволюционного семейства $\left\{w_{t, s}\right.$ : $0 \leqslant s \leqslant t \leqslant T\}$, то, выполняя замену переменной $t$ на $\tau$ посредством равенства $\tau=\ln \beta(t)$ и полагая $\Phi(z, \tau)=F(z, t(\tau))$, получим производящую функцию эволюционного семейства $\left\{\omega_{\tau, \theta}: 0 \leqslant \theta \leqslant \tau \leqslant \tau^{*}\right\}$, где $\tau^{*}=\ln \beta(T)$ и

$$
\omega_{\tau, \theta}(z)=\Phi^{-1}(\Phi(z, \theta), \tau)=w_{t(\tau), t(\theta)}(z) .
$$

При этом $\omega_{\tau, \theta}^{\prime}(1)=e^{\tau-\theta}, 0 \leqslant \theta \leqslant \tau \leqslant \tau^{*}$. Таким образом, для эволюционного семейства $\left\{\omega_{\tau, \theta}: 0 \leqslant \theta \leqslant \tau \leqslant \tau^{*}\right\}$ выполнены условия теоремы 1 и для почти всех $\tau$ будет выполняться равенство

$$
\frac{\partial}{\partial \tau} \omega_{\tau, \theta}(z)=-\omega_{\tau, \theta}(z)\left(1-\omega_{\tau, \theta}(z)\right) H\left(\omega_{\tau, \theta}(z), \tau\right),
$$

где $H(z, \tau)$ - инфинитезимальная производящая функция полугруппы $\mathfrak{L}[0,1]$ на промежутке $\left[0, \tau^{*}\right]$. 
Приведенные выше рассуждения показывают, что всякое эволюционное семейство в $\mathfrak{L}[0,1]$ с непрерывной функцией $\beta(t)=w_{t, 0}^{\prime}(1)$ можно преобразовать в нормированное эволюционное семейство так, чтобы выполнялось равенство $w_{t, s}^{\prime}(1)=e^{t-s}$, т.е. чтобы $\beta(t)=e^{t}$. При этом нормированное эволюционное семейство является решением эволюционного уравнения (3) с некоторой инфинитезимальной производящей функцией $H(z, t)$.

\section{§ 4. Эволюционное уравнение}

В $\S 3$ было показано, что нормированное эволюционное семейство в полугруппе $\mathfrak{L}[0,1]$ можно получить как решение эволюционного уравнения (3). В настоящем параграфе устанавливается, что каждая инфинитезимальная производящая функция порождает посредством уравнения (3) эволюционное семейство в полугруппе $\mathfrak{L}[0,1]$.

ДокАЗАТЕЛЬСтво тЕОРЕмЫ 2 . Пусть на $\mathbb{D} \times[0, T]$ определена комплекснозначная функция $H(z, t)$, которая голоморфна по $z$ в $\mathbb{D}$, измерима по $t$ на $[0, T]$ и такая, что $H(\cdot, t) \in \mathscr{Q}$ для почти всех $t$. Заметим, что решение дифференциального уравнения (3) с начальным условием $\left.w\right|_{t=s}=z$ эквивалентно решению интегрального уравнения

$$
\varphi(t, z)=z \exp \left\{-\int_{s}^{t}(1-\varphi(\tau, z)) H(\varphi(\tau, z), \tau) d \tau\right\},
$$

$s \leqslant t \leqslant T, z \in \mathbb{D}$. Для решения этого уравнения воспользуемся методом последовательных приближений. Положим $\varphi_{0}(t, z) \equiv z$ и для $n=1,2, \ldots$ определим

$$
\varphi_{n}(t, z)=z \exp \left\{-\int_{s}^{t}\left(1-\varphi_{n-1}(\tau, z)\right) H\left(\varphi_{n-1}(\tau, z), \tau\right) d \tau\right\} .
$$

Поскольку $H(\cdot, t) \in \mathscr{Q}$, то $\operatorname{Re}\{(1-z) H(z, t)\} \geqslant 0$ при $z \in \mathbb{D}$. Следовательно, $\varphi_{1}$, а также все последовательные приближения удовлетворяют неравенству $\left|\varphi_{n}(t, z)\right| \leqslant|z|, s \leqslant t \leqslant T, z \in \mathbb{D}$. Таким образом, последовательные приближения $\varphi_{n}(t, z), n=1,2, \ldots$, корректно определены, голоморфны по $z$ в $\mathbb{D}$ и абсолютно непрерывны по $t$ на $[s, T]$.

Покажем теперь, что последовательность функций $\varphi_{n}(t, z)$ при $n \rightarrow \infty$ сходится равномерно по $t$ на $[s, T]$ и локально равномерно по $z$ в $\mathbb{D}$. Замечая, что

$$
\left|e^{-\zeta_{1}}-e^{-\zeta_{2}}\right| \leqslant\left|\zeta_{1}-\zeta_{2}\right|
$$

для любых комплексных чисел $\zeta_{1}$ и $\zeta_{2}$ с неотрицательными вещественными частями, получаем

$$
\begin{aligned}
& \left|\varphi_{n+1}(t, z)-\varphi_{n}(t, z)\right| \\
& \quad \leqslant|z| \int_{s}^{t}\left|\left(1-\varphi_{n}(\tau, z)\right) H\left(\varphi_{n}(\tau, z), \tau\right)-\left(1-\varphi_{n-1}(\tau, z)\right) H\left(\varphi_{n-1}(\tau, z), \tau\right)\right| d \tau
\end{aligned}
$$


$n=1,2, \ldots$. Отсюда с использованием неравенства

$$
\left|\left(1-z^{\prime}\right) h\left(z^{\prime}\right)-\left(1-z^{\prime \prime}\right) h\left(z^{\prime \prime}\right)\right| \leqslant \frac{4\left|z^{\prime}-z^{\prime \prime}\right|}{\left(1-\left|z^{\prime}\right|\right)\left(1-\left|z^{\prime \prime}\right|\right)}
$$

$h \in \mathscr{Q}, z^{\prime}, z^{\prime \prime} \in \mathbb{D}$, которое устанавливается аналогично неравенству из леммы 1 , выводим

$$
\left|\varphi_{n+1}(t, z)-\varphi_{n}(t, z)\right| \leqslant \frac{4|z|}{(1-|z|)^{2}} \int_{s}^{t}\left|\varphi_{n}(\tau, z)-\varphi_{n-1}(\tau, z)\right| d \tau .
$$

В частности, при $n=1$ из этого неравенства следует

$$
\left|\varphi_{2}(t, z)-\varphi_{1}(t, z)\right| \leqslant \frac{4|z|}{(1-|z|)^{2}} \int_{s}^{t}\left|\varphi_{1}(\tau, z)-z\right| d \tau \leqslant \frac{2^{3}|z|^{2}}{(1-|z|)^{2}}(t-s) .
$$

Далее по индукции получаем

$$
\left|\varphi_{n+1}(t, z)-\varphi_{n}(t, z)\right| \leqslant \frac{2^{2 n+1}|z|^{n+1}}{(1-|z|)^{2 n}} \frac{(t-s)^{n}}{n !}
$$

$n=1,2, \ldots, s \leqslant t \leqslant T, z \in \mathbb{D}$.

Поскольку ряд

$$
\sum_{n=1}^{\infty} \frac{2^{2 n+1}|z|^{n+1}}{(1-|z|)^{2 n}} \frac{(t-s)^{n}}{n !}
$$

сходится равномерно по $t$ на $[s, T]$ и локально равномерно по $z$ в $\mathbb{D}$, то последовательность $\left\{\varphi_{n}(t, z)\right\}$ сходится равномерно по $t$ и локально равномерно по $z$ к некоторой функции $\varphi(t, z)$. Очевидно, что $\varphi(t, z)$ непрерывна по $t$ на $[s, T]$ и голоморфна по $z$ в $\mathbb{D}$. Кроме того, предельный переход в (8) при $n \rightarrow \infty$ показывает, что $\varphi(t, z)$ является решением интегрального уравнения $(7)$.

Таким образом, существование решения $w(t, z, s ; H)=\varphi(t, z)$ задачи Коши для дифференциального уравнения (3) доказано. Заметим также, что для дифференциального уравнения (3) выполнены условия теоремы единственности решения задачи Коши. Это доказывает однолистность отображения $w_{t, s}^{H}: z \mapsto w(t, z, s ; H)$ в единичном круге $\mathbb{D}$.

Остается показать, что отображения $w_{t, s}^{H}, s \leqslant t \leqslant t \leqslant T$, имеют конечную угловую производную в точке $z=1$ и для $\left(w_{t, s}^{H}\right)^{\prime}(1)=\varphi^{\prime}(t, 1)$ выполняется равенство (4). Для этого вначале покажем, что все итерации $\varphi_{n}(t, z), n=1,2, \ldots$, имеют конечную производную в точке $z=1$. В случае $n=0$ это очевидно, поскольку $\varphi_{0}(z) \equiv z$. Допустим теперь, что $\varphi_{n-1}(t, z)$ оставляет неподвижной точку $z=1$ и имеет в ней конечную угловую производную $\beta_{n-1}(t)=\varphi_{n-1}^{\prime}(t, 1)$, $s \leqslant t \leqslant T$. Если $z \in \Delta_{r}, 0 \leqslant r<1$, то в силу леммы 3 имеем $\varphi_{n-1}(t, z) \in \Delta_{\rho(t)}$, где

$$
\rho(t)=\frac{r \sqrt{\beta_{n-1}(t)+1}+\sqrt{\beta_{n-1}(t)-1}}{\sqrt{\beta_{n-1}(t)+1}+r \sqrt{\beta_{n-1}(t)-1}} .
$$

Но тогда из леммы 1 следует, что

$$
\angle \lim _{z \rightarrow 1}\left(1-\varphi_{n-1}(t, z)\right) H\left(\varphi_{n-1}(t, z), t\right)=0 .
$$


Отсюда и из равенства (8) получаем

$$
\angle \lim _{z \rightarrow 1} \varphi_{n}(t, z)=1,
$$

т.е. точка $z=1$ является неподвижной для функций $\varphi_{n}(t, \cdot), s \leqslant t \leqslant T$. Дифференцирование по $z$ равенства (8) приводит к соотношению

$$
\begin{aligned}
& \varphi_{n}^{\prime}(t, z)=\exp \left\{-\int_{s}^{t}\left(1-\varphi_{n-1}(\tau, z)\right) H\left(\varphi_{n-1}(\tau, z), \tau\right) d \tau\right\} \\
& \quad+\varphi_{n}(t, z) \int_{s}^{t}\left[H\left(\varphi_{n-1}(\tau, z), \tau\right)-\left(1-\varphi_{n-1}(\tau, z)\right) H^{\prime}\left(\varphi_{n-1}(\tau, z), \tau\right)\right] \varphi_{n-1}^{\prime}(\tau, z) d \tau .
\end{aligned}
$$

Из предыдущего следует, что угловой предел первого слагаемого в правой части последнего равенства существует и равен 1. Снова из леммы 1 получаем

$$
\angle \lim _{z \rightarrow 1}\left(1-\varphi_{n-1}(t, z)\right) H^{\prime}\left(\varphi_{n-1}(t, z), t\right)=0 .
$$

Таким образом,

$$
\beta_{n}(t)=\varphi_{n}^{\prime}(t, 1)=\angle \lim _{z \rightarrow 1} \varphi_{n}^{\prime}(t, z)=1+\int_{s}^{t} \beta_{n-1}(\tau) H(1, \tau) d \tau .
$$

Поскольку $H(1, t) \leqslant 1$, то для $\beta_{n}(t)$ выполняется неравенство

$$
\beta_{n}(t) \leqslant 1+\int_{s}^{t} \beta_{n-1}(\tau) d \tau .
$$

Отсюда с учетом равенства $\beta_{0}(t) \equiv 1$ следует

$$
\beta_{n}(t) \leqslant 1+(t-s)+\frac{1}{2 !}(t-s)^{2}+\cdots+\frac{1}{n !}(t-s)^{n} .
$$

Но тогда в силу леммы 2 получаем

$$
\beta(t)=\varphi^{\prime}(t, 1) \leqslant \underline{\lim }_{n \rightarrow \infty} \varphi_{n}^{\prime}(t, 1) \leqslant e^{t-s} .
$$

Итак, мы показали, что $w_{t, s}^{H}(z)=\varphi(t, z)$ является конформным отображением единичного круга в себя, оставляет неподвижной точку $z=1$ и имеет в ней конечную угловую производную, т.е. $w_{t, s}^{H} \in \mathfrak{L}[0,1]$. Для доказательства равенства (4) заметим, что дифференцирование по z соотношения (7) и последующий предельный переход при $z \rightarrow 1$ (внутри угловой области) приводит к интегральному уравнению

$$
\beta(t)=1+\int_{s}^{t} \beta(\tau) H(1, \tau) d \tau .
$$

Это означает, что $\beta(t)$ является решением дифференциального уравнения

$$
\frac{d \beta}{d t}=\beta H(1, t)
$$

с начальным условием $\left.\beta\right|_{t=s}=1$. Решение этой задачи Коши дает

$$
\beta(t)=\exp \left\{\int_{s}^{t} H(1, \tau) d \tau\right\},
$$

что эквивалентно равенству (4), и теорема 2 доказана. 


\section{$\S 5$. Теорема сходимости}

Для описания сходимости последовательностей инфинитезимальных производящих функций полугруппы $\mathfrak{L}[0,1]$, которая отвечает локально равномерной сходимости соответствующих последовательностей эволюционных семейств, введем следующие обозначения и определения.

Пусть $\mathfrak{F}(\mathbb{D},[0, T])$ - совокупность комплекснозначных функций $\Phi(z, t)$, определенных на $\mathbb{D} \times[0, T]$, голоморфных по $z$ в $\mathbb{D}$, измеримых по $t$ на $[0, T]$ и таких, что при всех $r \in(0,1)$ функция

$$
M_{r}(t ; \Phi)=\max _{|z| \leqslant r}|\Phi(z, t)|
$$

является суммируемой на $[0, T]$. Легко видеть, что $\mathfrak{F}(\mathbb{D},[0, T])$ представляет собой линейное пространство над полем комплексных чисел и если $\Phi \in$ $\mathfrak{F}(\mathbb{D},[0, T])$, то $\Phi^{\prime}(z, t)=\partial \Phi(z, t) / \partial z$ также принадлежит $\mathfrak{F}(\mathbb{D},[0, T])$. Кроме того, интеграл $\int_{[0, T]} \Phi(z, t) d t$ является аналитической в $\mathbb{D}$ функцией.

Будем говорить, что последовательность $\left\{\Phi_{n}\right\} \subset \mathfrak{F}(\mathbb{D},[0, T])$ слабо сходится в $\mathfrak{F}(\mathbb{D},[0, T])$ к функции $\Phi(z, t)$, если для любой ограниченной измеримой на $[0, T]$ функции $\eta(t)$

$$
\int_{[0, T]} \eta(t) \Phi_{n}(z, t) d t \rightarrow \int_{[0, T]} \eta(t) \Phi(z, t) d t
$$

локально равномерно в $\mathbb{D}$ при $n \rightarrow \infty$. Слабая сходимость в $\mathfrak{F}(\mathbb{D},[0, T])$ была введена и изучалась в работах [23], [24]. В частности, в [23] был установлен принцип компактности, который состоит в следующем. Пусть последовательность функций $\Phi_{n}(z, t), n=1,2, \ldots$, из $\mathfrak{F}(\mathbb{D},[0, T])$ такова, что для каждого $r \in(0,1)$ найдется такая суммируемая на $[0, T]$ функция $N_{r}(t)$, что $M_{r}\left(t ; \Phi_{n}\right) \leqslant N_{r}(t)$ для всех $n=1,2, \ldots$ и почти всех $t \in[0, T]$. Тогда из этой последовательности можно выделить подпоследовательность, слабо сходящуюся к некоторой функции $\Phi(z, t)$ в $\mathfrak{F}(\mathbb{D},[0, T])$. При этом для почти всех $t \in[0, T]$ функция $\Phi(\cdot, t)$ должна принадлежать замкнутой выпуклой оболочке последовательности $\left\{\Phi_{n}(\cdot, t)\right\}$ в пространстве $\mathscr{H}(\mathbb{D})$ всех голоморфных в $\mathbb{D}$ функций, наделенном топологией локально равномерной сходимости.

Заметим, что всякая инфинитезимальная производящая функция $H(z, t)$ на $[0, T]$ полугруппы $\mathfrak{L}[0,1]$ принадлежит пространству $\mathfrak{F}(\mathbb{D},[0, T])$. Это следует из неравенства

$$
|h(z)| \leqslant \frac{2}{1-|z|},
$$

которое имеет место для любой функции $h \in \mathscr{Q}$ и $z \in \mathbb{D}$.

ТЕОрема 6. Пусть $\left\{H_{n}\right\}$ - последовательность инфинитезимальных производящих функций полугруппы $\mathfrak{L}[0,1]$ на промежутке $[0, T], T>0, u H_{n} \rightarrow H$ слабо в $\mathfrak{F}(\mathbb{D},[0, T])$ при $n \rightarrow \infty$. Тогда предельная функиия $H(z, t)$ также будет инфинитезимальной производящей функцией полугруппь $\mathfrak{L}[0,1]$ u $w_{t, s}^{H_{n}}(z) \rightarrow$ $w_{t, s}^{H}(z)$ локально равномерно в $\mathbb{D} n$ ри $n \rightarrow \infty$ для всех $s, t, 0 \leqslant s \leqslant t \leqslant T$. 
ДокАЗАТЕльство. Поскольку класс $\mathscr{Q}$ является компактным выпуклым множеством в пространстве $\mathscr{H}(\mathbb{D})$, то, как следует из свойств слабой сходимости в $\mathfrak{F}(\mathbb{D},[0, T])$ (см. [23]), предельная функция $H(z, t)$ удовлетворяет условию $H(\cdot, t) \in \mathscr{Q}$ для почти всех $t$, т.е. $H(z, t)$ является инфинитезимальной производящей функцией полугруппы $\mathfrak{L}[0,1]$ на промежутке $[0, T]$.

Фиксируем произвольно $z \in \mathbb{D}$ и $s \in[0, T)$. Обозначим для удобства

$$
\zeta_{n}(t)=w_{t, s}^{H_{n}}(z), \quad s \leqslant t \leqslant T,
$$

и покажем, что $\zeta_{n}(t) \rightarrow w_{t, s}^{H}(z)$ равномерно на $[s, T]$ при $n \rightarrow \infty$. Заметим, что в силу леммы Шварца

$$
\left|\zeta_{n}(t)\right|=\left|w_{t, s}^{H_{n}}(z)\right| \leqslant|z|
$$

для всех $t \in[s, T]$ и $n=1,2, \ldots$. Далее, для любых $t^{\prime}, t^{\prime \prime}, s \leqslant t^{\prime}<t^{\prime \prime} \leqslant T$, в силу неравенства из леммы 4 получаем

$$
\begin{aligned}
\left|\zeta_{n}\left(t^{\prime \prime}\right)-\zeta_{n}\left(t^{\prime}\right)\right| & =\left|w_{t^{\prime \prime}, t^{\prime}}^{H_{n}} \circ w_{t^{\prime}, s}^{H_{n}}(z)-w_{t^{\prime}, s}^{H_{n}}(z)\right| \leqslant \frac{4\left|w_{t^{\prime}, s}^{H_{n}}(z)\right|}{1-\left|w_{t^{\prime}, s}^{H_{n}}(z)\right|}\left(\left(w_{t^{\prime \prime}, t^{\prime}}^{H_{n}}\right)^{\prime}(1)-1\right) \\
& \leqslant \frac{4|z|}{1-|z|}\left(e^{t^{\prime \prime}-t^{\prime}}-1\right) \leqslant \frac{4|z| e^{T}}{1-|z|}\left|t^{\prime \prime}-t^{\prime}\right| .
\end{aligned}
$$

Таким образом, последовательность функций $\zeta_{n}(t), n=1,2, \ldots$, является равномерно ограниченной и равностепенно непрерывной на отрезке $[s, T]$. В силу теоремы Арцела-Асколи равномерная сходимость $\zeta_{n}(t) \rightarrow w_{t, s}^{H}(z)$ на $[s, T]$ будет установлена, если показать, что любая равномерно сходящаяся на $[s, T]$ подпоследовательность из $\left\{\zeta_{n}\right\}$ имеет своим пределом функцию $t \mapsto w_{t, s}^{H}(z)$, $s \leqslant t \leqslant T$.

Чтобы не усложнять обозначений, допустим, что сама последовательность $\left\{\zeta_{n}\right\}$ сходится равномерно на $[s, T]$ к некоторой функции $\zeta(t)$. Нам нужно показать, что $\zeta(t)=w_{t, s}^{H}(z)$ при всех $t \in[s, T]$. Это равенство эквивалентно тому, что предельная функция $\zeta(t)$ является решением интегрального уравнения

$$
\zeta(t)=z \exp \left\{-\int_{s}^{t}(1-\zeta(\tau)) H(\zeta(\tau), \tau) d \tau\right\}
$$

$s \leqslant t \leqslant T$. Замечая теперь, что при каждом $n=1,2, \ldots$ выполняется равенство

$$
\zeta_{n}(t)=z \exp \left\{-\int_{s}^{t}\left(1-\zeta_{n}(\tau)\right) H_{n}\left(\zeta_{n}(\tau), \tau\right) d \tau\right\}
$$

получаем, что доказательство того, что $\zeta(t)$ является решением интегрального уравнения (10), будет следовать из предельного соотношения

$$
\lim _{n \rightarrow \infty} \int_{s}^{t}\left(1-\zeta_{n}(\tau)\right) H_{n}\left(\zeta_{n}(\tau), \tau\right) d \tau=\int_{s}^{t}(1-\zeta(\tau)) H(\zeta(\tau), \tau) d \tau
$$


Считая $s$ и $t$ фиксированными, заметим, что

$$
\begin{aligned}
\int_{s}^{t}(1-\zeta(\tau)) H(\zeta(\tau), \tau) d \tau-\int_{s}^{t}\left(1-\zeta_{n}(\tau)\right) H_{n}\left(\zeta_{n}(\tau), \tau\right) d \tau \\
=\int_{s}^{t}\left(\zeta_{n}(\tau)-\zeta(\tau)\right) H_{n}(\zeta(\tau), \tau) d \tau \\
\quad+\int_{s}^{t}\left(1-\zeta_{n}(\tau)\right)\left(H_{n}(\zeta(\tau), \tau)-H_{n}\left(\zeta_{n}(\tau), \tau\right)\right) d \tau \\
\quad+\int_{s}^{t}(1-\zeta(\tau))\left(H(\zeta(\tau), \tau)-H_{n}(\zeta(\tau), \tau)\right) d \tau
\end{aligned}
$$

и покажем, что каждый из интегралов в правой части последнего равенства стремится к нулю при $n \rightarrow \infty$. Вместе с этим будет доказано равенство (11), что эквивалентно сходимости $w_{t, s}^{H_{n}}(z) \rightarrow w_{t, s}^{H}(z)$ при $n \rightarrow \infty$ для всех $z \in \mathbb{D}$.

Стремление к нулю первых двух интегралов при $n \rightarrow \infty$ следует непосредственно из равномерной сходимости последовательности функций $\zeta_{n}(t)$ на $[s, T]$ и оценок в классе $\mathscr{Q}$. Действительно, используя неравенство (9), получаем

$$
\left|\int_{s}^{t}\left(\zeta_{n}(\tau)-\zeta(\tau)\right) H_{n}(\zeta(\tau), \tau) d \tau\right| \leqslant \frac{2}{1-|z|} \int_{s}^{t}\left|\zeta_{n}(\tau)-\zeta(\tau)\right| d \tau \rightarrow 0
$$

при $n \rightarrow \infty$. Применение неравенства из леммы 1 приводит к следующему результату:

$$
\begin{aligned}
& \left|\int_{s}^{t}\left(1-\zeta_{n}(\tau)\right)\left(H_{n}(\zeta(\tau), \tau)-H_{n}\left(\zeta_{n}(\tau), \tau\right)\right) d \tau\right| \\
& \quad \leqslant \int_{s}^{t}\left|1-\zeta_{n}(\tau)\right| \frac{2\left|\zeta(\tau)-\zeta_{n}(\tau)\right|}{(1-|\zeta(\tau)|)\left(1-\left|\zeta_{n}(\tau)\right|\right)} d \tau \\
& \quad \leqslant \frac{2(1+|z|)}{(1-|z|)^{2}} \int_{s}^{t}\left|\zeta_{n}(\tau)-\zeta(\tau)\right| d \tau \rightarrow 0
\end{aligned}
$$

при $n \rightarrow \infty$. Для доказательства предельного соотношения

$$
\lim _{n \rightarrow \infty} \int_{s}^{t}(1-\zeta(\tau))\left(H(\zeta(\tau), \tau)-H_{n}(\zeta(\tau), \tau)\right) d \tau=0
$$

фиксируем произвольно $\varepsilon>0$ и выполним разбиение $s=\tau_{0}<\tau_{1}<\cdots<\tau_{m}=t$ так, чтобы неравенство $\left|\zeta\left(\tau^{\prime}\right)-\zeta\left(\tau^{\prime \prime}\right)\right|<\varepsilon$ выполнялось для любых $\tau^{\prime}, \tau^{\prime \prime}$ из одного и того же интервала $\left[\tau_{k-1}, \tau_{k}\right], k=1, \ldots, m$. Это можно сделать в силу равномерной непрерывности функции $\zeta(t)$ на $[s, t]$. Введем кусочно постоянную функцию $\xi(\tau)=\zeta\left(\tau_{k}\right)$ при $\tau \in\left(\tau_{k-1}, \tau_{k}\right], k=1, \ldots, m$. В силу сделанных предположений относительно разбиения для всех $\tau \in[s, t]$ будет выполняться неравенство $|\zeta(\tau)-\xi(\tau)|<\varepsilon$. Отсюда с использованием неравенства из леммы 1 получаем

$$
\begin{aligned}
|H(\zeta(\tau), \tau)-H(\xi(\tau), \tau)| & \leqslant \frac{2|\zeta(\tau)-\xi(\tau)|}{(1-|\zeta(\tau)|)(1-|\xi(\tau)|)} \leqslant \frac{2 \varepsilon}{(1-|z|)^{2}} \\
\left|H_{n}(\zeta(\tau), \tau)-H_{n}(\xi(\tau), \tau)\right| & \leqslant \frac{2 \varepsilon}{(1-|z|)^{2}}
\end{aligned}
$$


для всех $\tau \in[s, t]$. Но тогда

$$
\begin{aligned}
& \left|\int_{s}^{t}(1-\zeta(\tau))\left(H(\zeta(\tau), \tau)-H_{n}(\zeta(\tau), \tau)\right) d \tau\right| \\
& \leqslant\left|\int_{s}^{t}(1-\zeta(\tau))(H(\zeta(\tau), \tau)-H(\xi(\tau), \tau)) d \tau\right| \\
& \quad+\left|\int_{s}^{t}(1-\zeta(\tau))\left(H(\xi(\tau), \tau)-H_{n}(\xi(\tau), \tau)\right) d \tau\right| \\
& \quad+\left|\int_{s}^{t}(1-\zeta(\tau))\left(H_{n}(\xi(\tau), \tau)-H_{n}(\zeta(\tau), \tau)\right) d \tau\right| \\
& \leqslant \frac{4 \varepsilon(t-s)(1+|z|)}{(1-|z|)^{2}}+\left|\int_{s}^{t}(1-\zeta(\tau))\left(H(\xi(\tau), \tau)-H_{n}(\xi(\tau), \tau)\right) d \tau\right|
\end{aligned}
$$

Поскольку $H_{n} \rightarrow H$ слабо в $\mathfrak{F}(\mathbb{D},[0, T])$, то имеем также

$$
\begin{aligned}
\int_{s}^{t}(1-\zeta(\tau))\left(H(\xi(\tau), \tau)-H_{n}(\xi(\tau), \tau)\right) d \tau \\
\quad=\sum_{k=1}^{m} \int_{\tau_{k-1}}^{\tau_{k}}(1-\zeta(\tau))\left(H\left(\zeta\left(\tau_{k}\right), \tau\right)-H_{n}\left(\zeta\left(\tau_{k}\right), \tau\right)\right) d \tau \rightarrow 0
\end{aligned}
$$

при $n \rightarrow \infty$. Отсюда и из того, что $\varepsilon>0$ выбиралось произвольно, следует предельное соотношение (12).

Таким образом, $w_{t, s}^{H_{n}}(z) \rightarrow w_{t, s}^{H}(z)$ при $n \rightarrow \infty$ для любого $z \in \mathbb{D}$. Но тогда в силу теоремы Витали (см., например, [25]) $w_{t, s}^{H_{n}}(z) \rightarrow w_{t, s}^{H}(z)$ локально равномерно в $\mathbb{D}$ при $n \rightarrow \infty$.

Теорема 6 доказана.

\section{§ 6. Теорема вложения}

Начнем этот параграф с доказательства теоремы 3, которая играет основополагающую роль в развитии параметрического метода. После этого изучим структуру полугруппы $\mathfrak{L}[0,1]$ и ее аппроксимационные свойства.

ДоКАЗАТЕЛЬСтво тЕОРЕмы 3. Выделим для удобства в доказательстве теоремы вложения три пункта.

1. Аппроксимация отображениями на области специального вида. Вначале покажем, что любую функцию из $\mathfrak{L}[0,1]$ можно представить как локально равномерный предел последовательности функций из $\mathfrak{L}[0,1]$, каждая из которых отображает $\mathbb{D}$ на область, полученную удалением из $\mathbb{D}$ конечного числа аналитических жордановых дуг. Пусть $f \in \mathfrak{L}[0,1]$ и $f^{\prime}(1)=\beta>1$. Для $r \in(0,1)$ рассмотрим дробно-линейное преобразование

$$
L_{r}(z)=\frac{2 r z}{1+r-(1-r) z},
$$

оображающее единичный круг $\mathbb{D}$ на орицикл $\mathbb{H}_{\alpha(r)}$, где $\alpha(r)=(1+r) /(1-r)$. При этом $L_{r}(0)=0, L_{r}(1)=1$ и $L_{r}^{\prime}(1)=(1+r) /(2 r)$. Следовательно, функция $f_{r}=f \circ L_{r}$ принадлежит $\mathfrak{L}[0,1]$ и $f_{r}^{\prime}(1)=\beta(1+r) /(2 r)$. Заметим, что 
$f_{r}^{\prime}(1) \rightarrow \beta$ и $f_{r}(z) \rightarrow f(z)$ локально равномерно в $\mathbb{D}$ при $r \rightarrow 1$. Поэтому достаточно показать, что каждую функцию $f_{r}$ можно представить в виде предела последовательности конформных отображений на области с разрезами.

Функция $f_{r}, 0<r<1$, отображает единичный круг $\mathbb{D}$ на область $D_{r}=$ $f\left(\mathbb{H}_{\alpha(r)}\right)$, граница которой представляет собой замыкание аналитической жордановой дуги $\Gamma_{r}$ с параметризацией

$$
\Gamma_{r}: w(t)=f\left(\zeta_{r}(t)\right), \quad 0<t<2 \pi
$$

где

$$
\zeta_{r}(t)=\frac{1}{2}\left(1-r+(1+r) e^{i t}\right)
$$

В силу теоремы ЖКюлиа-Каратеодори дуга $\Gamma_{r}$ расположена в орицикле $\mathbb{H}_{\beta \alpha(r)}$, и потому ее замыкание имеет единственную общую точку $z=1$ с единичной окружностью $\mathbb{T}$. Таким образом, $\mathbb{T}$ и $\Gamma_{r}$ ограничивают некоторую область $G_{r} \subset \mathbb{D}$, в которой можно провести сечение в виде ломаной $\Lambda_{r}$ без самопересечений, соединяющей граничные точки $w=-1$ и $w=f(-r)$.

Выберем теперь монотонно убывающую последовательность $\left\{t_{n}\right\}$, удовлетворяющую условиям $t_{1}<\pi / 2$ и $t_{n} \searrow 0$ при $n \rightarrow \infty$. Для каждого $n=1,2, \ldots$ через $\Gamma_{r, n}$ обозначим аналитическую жорданову дугу с параметризацией

$$
\Gamma_{r, n}: w(t)=f\left(\zeta_{r}(t)\right), \quad t_{n} \leqslant t \leqslant 2 \pi-t_{n} .
$$

Пусть $D_{r, n}$ - область, которая получается удалением из единичного круга $\mathbb{D}$ ломаной $\Lambda_{r}$ и дуги $\Gamma_{r, n}$. Как следует из построения, области $D_{r, n}$ являются односвязными, содержат точку $w=0$, монотонно убывают: $D_{r, 1} \supset D_{r, 2} \supset \cdots$, и $D_{r, n} \rightarrow D_{r}$ в смысле сходимости к ядру при $n \rightarrow \infty$. Кроме того, для каждой области $D_{r, n}$ проколотая единичная окружность $\mathbb{T} \backslash\{-1\}$ является свободной граничной дугой, т.е. ни одна из ее точек не является предельной для множества $\Lambda_{r} \cup \Gamma_{r, n}$.

Обозначим через $f_{n}$ конформное отображение единичного круга $\mathbb{D}$ на область $D_{r, n}$, нормированное условиями $f_{n}(0)=0, f_{n}(1)=1$. Заметим, что открытая дуга $\lambda_{n}=f_{n}^{-1}(\mathbb{T} \backslash\{-1\})$ расположена на единичной окружности и содержит $z=1$ в качестве внутренней точки. В силу принципа симметрии Римана-Шварца $f_{n}$ аналитически продолжается через дугу $\lambda_{n}$ во внешность единичного круга. Таким образом, $f_{n} \in \mathfrak{L}[0,1]$ и $f_{n}(z) \rightarrow f_{r}(z)$ локально равномерно в $\mathbb{D}$ при $n \rightarrow \infty$. Кроме того, в силу вложенности областей $D_{r, 1} \supset D_{r, 2} \supset \cdots$ числовая последовательность $\left\{f_{n}^{\prime}(1)\right\}$ является монотонно возрастающей, и на основе леммы 2 имеем $f_{n}^{\prime}(1) \nearrow f_{r}^{\prime}(1)$ при $n \rightarrow \infty$.

2. Вложение для конформных отображений специального вида. Пусть $f$ принадлежит классу $\mathfrak{L}[0,1]$ и отображает единичный круг на область $D=\mathbb{D} \backslash \Gamma$, где $\Gamma$ представляет собой объединение жордановой ломаной $\Lambda$, соединяющей точки $w=-1$ и $w^{*} \in \mathbb{D}$, и двух аналитических жордановых дуг $\Gamma_{1}$ и $\Gamma_{2}$, которые имеют одну общую концевую точку $w^{*}$ и не имеют других общих точек ни между собой, ни с ломаной $\Lambda$. При этом на $\mathbb{T} \backslash\{-1\}$ нет ни одной предельной точки множества Г. Вытирая последовательно дуги $\Gamma_{1}, \Gamma_{2}$ и ломаную $\Lambda$, 
получим семейство односвязных областей $D_{t}, 0 \leqslant t \leqslant T$, удовлетворяющее условиям:

a) $D_{0}=D, D_{T}=\mathbb{D}$;

b) $D_{t^{\prime}} \subset D_{t^{\prime \prime}}$ при $0 \leqslant t^{\prime}<t^{\prime \prime} \leqslant T$;

c) $D_{t} \rightarrow D_{t_{0}}$ в смысле сходимости к ядру при $t \rightarrow t_{0}$.

Пусть $w=F(z, t)$ - конформное отображение единичного круга $\mathbb{D}$ на область $D_{t}, 0 \leqslant t \leqslant T$, с нормировкой $F(0, t)=0, F(1, t)=1$. Поскольку $\mathbb{T} \backslash\{-1\}$ является свободной открытой дугой границы области $D_{t}$, то $F(z, t)$ аналитически продолжается во внешность единичного круга через некоторую дугу единичной окружности, содержащую $z=1$ в качестве внутренней точки. Поэтому в силу условий b) и с) $\gamma(t)=F^{\prime}(1, t)$ является непрерывной строго монотонно убывающей функцией. Используя замену переменной, если необходимо, можно добиться выполнения равенства $\gamma(t)=e^{T-t}$. При этом в силу условия а) $\gamma(0)=e^{T}=f^{\prime}(1)$ и $\gamma(T)=1$. Таким образом, $F(z, t)$ является производящей функцией эволюционного семейства

$$
\left\{w_{t, s}(z)=F^{-1}(F(z, s), t): 0 \leqslant s \leqslant t \leqslant T\right\}
$$

полугруппы $\mathfrak{L}[0,1]$. Из перечисленных выше свойств производящей функции $F(z, t)$ следует, что $w_{T, 0}(z)=f(z)$ и

$$
\beta(t)=w_{t, 0}^{\prime}(1)=\frac{\gamma(0)}{\gamma(t)}=e^{t},
$$

$0 \leqslant t \leqslant T$. Поэтому по теореме 1 найдется такая инфинитезимальная производящая функция $H(z, t)$ полугруппы $\mathfrak{L}[0,1]$, что $w_{t, s}(z)=w_{t, s}^{H}(z), 0 \leqslant s \leqslant t \leqslant T$. В частности, $w_{T, 0}^{H}(z)=f(z)$.

3. Общий случай. Пусть $f$ - произвольная функция из $\mathfrak{L}[0,1]$ и $f^{\prime}(1)=\beta>0$. Как следует из приведенных выше рассуждений, найдется такая последовательность функций $f_{n}, n=1,2, \ldots$, из $\mathfrak{L}[0,1]$, что $f_{n}^{\prime}(1) \rightarrow \beta, f_{n}(z) \rightarrow f(z)$ локально равномерно в $\mathbb{D}$ при $n \rightarrow \infty$ и для каждого $n=1,2, \ldots$ имеет место представление

$$
f_{n}(z)=w_{T_{n}, 0}^{H_{n}}(z),
$$

где $T_{n}=\ln f_{n}^{\prime}(1)$, а $H_{n}(z, t)$ - инфинитезимальная производящая функция полугруппы $\mathfrak{L}[0,1]$ на промежутке $\left[0, T_{n}\right]$. Определим $T^{*}=\sup \left\{T_{n}: n=1,2, \ldots\right\}$ и заметим, что $\ln \beta=T \leqslant T^{*}$, поскольку $f_{n}^{\prime}(1) \rightarrow \beta$. Все функции $H_{n}(z, t)$ будем рассматривать как элементы пространства $\mathfrak{F}\left(\mathbb{D},\left[0, T^{*}\right]\right)$, полагая $H_{n}(z, t)=0$ при $t \in\left(T_{n}, T^{*}\right]$. При этом будут выполняться равенства

$$
f_{n}(z)=w_{T^{*}, 0}^{H_{n}}(z)
$$

поскольку $w_{t, s}^{H_{n}}(z) \equiv z$ при $T_{n} \leqslant s \leqslant t \leqslant T^{*}$.

В силу принципа компактности для слабой сходимости в пространстве $\mathfrak{F}\left(\mathbb{D},\left[0, T^{*}\right]\right)$ из последовательности $\left\{H_{n}\right\}$ можно выделить подпоследовательность $\left\{H_{n_{k}}\right\}$, которая слабо сходится в $\mathfrak{F}\left(\mathbb{D},\left[0, T^{*}\right]\right)$ к некоторой $\mathscr{Q}$-значной 
функции $H(z, t)$. Другими словами, $H(z, t)$ - инфинитезимальная производящая функция полугруппы $\mathfrak{L}[0,1]$. Чтобы не усложнять обозначений, будем считать, что $H_{n} \rightarrow H$ слабо в $\mathfrak{F}\left(\mathbb{D},\left[0, T^{*}\right]\right)$. Но тогда по теореме сходимости $w_{t, s}^{H_{n}}(z) \rightarrow w_{t, s}^{H}(z)$ локально равномерно в $\mathbb{D}$ при $n \rightarrow \infty$ для всех $s, t$, $0 \leqslant s \leqslant t \leqslant T^{*}$. В частности,

$$
f(z)=\lim _{n \rightarrow \infty} f_{n}(z)=\lim _{n \rightarrow \infty} w_{T^{*}, 0}^{H_{n}}(z)=w_{T^{*}, 0}^{H}(z) .
$$

Далее, если $t \in\left(T, T^{*}\right]$, то для достаточно больших номеров $n$ будет выполняться неравенство $T_{n}<t$ и, следовательно, $w_{T^{*}, t}^{H_{n}}(z) \equiv z$ в $\mathbb{D}$. Это означает, что $w_{T^{*}, t}^{H}(z) \equiv z$ в $\mathbb{D}$ при всех $t \in\left[T, T^{*}\right]$. Но тогда

$$
f(z)=w_{T^{*}, 0}^{H}(z)=w_{T^{*}, T}^{H} \circ w_{T, 0}^{H}(z)(z)=w_{T, 0}^{H}(z) .
$$

Теорема 3 доказана.

Аналогом уравнения Лёвнера для полугруппы $\mathfrak{L}[0,1]$ можно считать следующее:

$$
\frac{d w}{d t}=-w(1-w) \frac{1-\varkappa(t)}{1-\varkappa(t) w},
$$

где $\varkappa(t)$ - непрерывная унимодулярная (т.е. удовлетворяющая условию $|\varkappa(t)|=1)$ функция. В частности, совокупность функций, вложимых в эволюционные семейства, которые воспроизводятся этим уравнением, образуют плотное в $\mathfrak{L}[0,1]$ множество. Это следует из теорем 3,6 и того, что функции вида $H(z, t)=(1-\varkappa(t)) /(1-\varkappa(t) z)$, где $\varkappa(t)$ - непрерывная унимодулярная на $[0, T]$ функция, образуют плотное в смысле слабой сходимости в $\mathfrak{F}(\mathbb{D},[0, T])$ подмножество множества всех инфинитезимальных производящих функций полугруппы $\mathfrak{L}[0,1]$ на промежутке $[0, T]$.

Введем также в рассмотрение семейство функций, которые для полугруппы $\mathfrak{L}[0,1]$ играют роль, аналогичную той, которую для полугруппы $\mathfrak{L}$ играет функция Кёбе. Пусть $\sigma=e^{i \theta},-\pi / 2<\theta<\pi / 2$, и определим

$$
K_{\theta}(z)=\frac{z}{(1-z)^{1+\sigma^{2}}},
$$

где под степенной функцией $(1-z)^{1+\sigma^{2}}$ понимается непрерывная ветвь, принимающая значение 1 при $z=0$. Для более удобных вычислений, связанных с выбором ветвей при дифференцировании функции $K_{\theta}$, следует записать ее представление в виде

$$
K_{\theta}(z)=z \exp \left\{-\left(1+\sigma^{2}\right) \ln (1-z)\right\}
$$

где под $\ln (1-z)$ понимается ветвь в $\mathbb{D}$, обращающаяся в нуль при $z=0$. Тогда

$$
K_{\theta}^{\prime}(z)=\left(1+\left(1+\sigma^{2}\right) \frac{z}{1-z}\right) \exp \left\{-\left(1+\sigma^{2}\right) \ln (1-z)\right\}
$$

и

$$
\operatorname{Re}\left\{\bar{\sigma} \frac{z K_{\theta}^{\prime}(z)}{K_{\theta}(z)}\right\}=\operatorname{Re}\left\{\bar{\sigma}+(\bar{\sigma}+\sigma) \frac{z}{1-z}\right\}=\cos \theta\left(1+2 \operatorname{Re} \frac{z}{1-z}\right)>0
$$


при $z \in \mathbb{D}$. Это означает, что $K_{\theta}$ является $\theta$-спиральной функцией (см., например, [26]). Более точно, $K_{\theta}$ является голоморфной и однолистной в единичном круге $\mathbb{D}$ функцией и отображает его на дополнение дуги логарифмической спирали

$$
w(t)=-e^{\sigma t}, \quad t \geqslant t^{*}(\theta)=-2 \cos \theta[\theta \operatorname{tg} \theta+\ln (2 \cos \theta)] .
$$

Заметим, что $K_{\theta}$ совпадает с функцией Кёбе при $\theta=0$ и $K_{\theta}(z) \rightarrow z$ локально равномерно в $\mathbb{D}$ при $\theta \rightarrow \pm \pi / 2$.

Из свойств функции $K_{\theta},-\pi / 2<\theta<\pi / 2$, следует, что для любого $\tau>0$ корректно определена функция

$$
\phi_{\theta}^{\tau}(z)=K_{\theta}^{-1}\left(e^{-\tau \sigma} K_{\theta}(z)\right)
$$

которая конформно отображает единичный круг $\mathbb{D}$ на область, получаемую удалением из $\mathbb{D}$ аналитической дуги $\Lambda_{\theta, \tau}=K_{\theta}^{-1}\left(\Gamma_{\theta, \tau}\right)$, где $\Gamma_{\theta, \tau}$ - часть логарифмической спирали:

$$
w(t)=-e^{\sigma t}, \quad t^{*}(\theta)-\tau \leqslant t \leqslant t^{*}(\theta) .
$$

Легко видеть, что $\tau \mapsto \phi_{\theta}^{\tau}$ является однопараметрической полугруппой с инфинитезимальной образующей

$$
\left.\frac{\partial}{\partial \tau} \phi_{\theta}^{\tau}(z)\right|_{\tau=0}=-\frac{\sigma K_{\theta}(z)}{K_{\theta}^{\prime}(z)}=-\frac{1}{2 \cos \theta} z(1-z) \frac{1-\varkappa}{1-\varkappa z},
$$

где $\varkappa=-\sigma^{2}$. Другими словами, $\phi_{\theta}^{\tau}(z)=w_{\alpha \tau, 0}^{H}(z)$, где $\alpha=1 /(2 \cos \theta)$, а инфинитезимальная производящая функция $H$ имеет вид

$$
H(z, t)=\frac{1-\varkappa}{1-\varkappa z} .
$$

В силу теоремы 2 отображение $\phi_{\theta}^{\tau}$ принадлежит $\mathfrak{L}[0,1]$ и

$$
\left(\phi_{\theta}^{\tau}\right)^{\prime}(1)=\exp \left\{\int_{0}^{\alpha \tau} H(1, t) d t\right\}=\exp \left\{\frac{\tau}{2 \cos \theta}\right\} .
$$

В принятых выше обозначениях имеет место следующий результат.

Tеорема 7. Совокупность функций вида

$$
\phi(z)=\phi_{\theta_{1}}^{\tau_{1}} \circ \cdots \circ \phi_{\theta_{n}}^{\tau_{n}}(z),
$$

где $\tau_{k}>0, \theta_{k} \in(-\pi / 2, \pi / 2), k=1, \ldots, n, n=1,2, \ldots$, образует плотное в $\mathfrak{L}[0,1]$ подмножество относительно топологии локально равномерной в $\mathbb{D}$ сходимости.

ДоказАтельство. Пусть $f \in \mathfrak{L}[0,1]$ и $f^{\prime}(1)=\beta>1$. В силу теоремы 3 найдется такая инфинитезимальная производящая функция $H(z, t)$ на промежутке $[0, T], T=\ln \beta$, что $f(z)=w_{T, 0}^{H}(z)$. Заметим, что класс $\mathscr{Q}$ является 
компактным выпуклым множеством в пространстве $\mathscr{H}(\mathbb{D})$ и множество его крайних точек составляют функции

$$
g_{\varkappa}(z)=\frac{1-\varkappa}{1-\varkappa z}
$$

$\varkappa \in \mathbb{T}, g_{1}(z) \equiv 0$. Поэтому в силу теоремы 8 из [27] найдется последовательность инфинитезимальных производящих функций $H_{j}(z, t), j=1,2, \ldots$, вида

$$
H_{j}(z, t)=g_{\varkappa_{j, k}}(z), \quad \frac{k-1}{n_{j}} T<t \leqslant \frac{k}{n_{j}} T,
$$

$k=1, \ldots, n_{j}, \varkappa_{j, k} \in \mathbb{T} \backslash\{1\}$, такая, что $H_{j} \rightarrow H$ слабо в $\mathfrak{F}(\mathbb{D},[0, T])$ при $j \rightarrow \infty$. Но тогда в силу теоремы 6 имеем $w_{T, 0}^{H_{j}}(z) \rightarrow w_{T, 0}^{H}(z)=f(z)$ локально равномерно в $\mathbb{D}$ при $j \rightarrow \infty$. Остается заметить, что для каждого $j=1,2, \ldots$ отображение $w_{T, 0}^{H_{j}}$ можно представить в виде композиции

$$
w_{T, 0}^{H_{j}}=\psi_{n_{j}} \circ \cdots \circ \psi_{1}(z),
$$

в которой каждая функция $\psi_{k}, k=1, \ldots, n_{j}$, имеет требуемый вид

$$
\psi_{k}(z)=\phi_{\theta_{k}}^{\tau_{k}}(z)
$$

где $\theta_{k} \in(-\pi / 2, \pi / 2)$ и $e^{i 2 \theta_{k}}=-\varkappa_{j, k}$, а $\tau_{k}=2 T\left(\cos \theta_{k}\right) / n_{j}$. Теорема доказана.

Интересно сравнить этот результат с аналогичным для полугруппы $\mathfrak{L}$ (см. $[3],[24])$.

\section{§ 7. Параметрический метод и угловая производная}

Возможность вложения отображения в эволюционное семейство, которое дифференцируемо и воспроизводится посредством эволюционного уравнения, позволяет экстремальные задачи, определенные на классе таких отображений, перевести на класс соответствующих инфинитезимальных производящих функций. Другими словами, задача об экстремальном конформном отображении может быть сформулирована как задача оптимального управления, где роль управления играет инфинитезимальная производящая функция. Инфинитезимальное описание полугруппы $\mathfrak{L}$ позволило решить ряд трудных внутренних задач теории однолистных функций (см. [5]). Теорема 3 об инфинитезимальном описании полугруппы $\mathfrak{L}[0,1]$ позволяет расширить возможности параметрического метода на экстремальные задачи конформного отображения, включающие угловую производную в неподвижной точке на границе единичного круга.

ДокаЗАТЕЛЬСтво теоремы 4. Пусть $f \in \mathfrak{L}[0,1]$ и $f^{\prime}(1)=\beta>1$. Тогда, как следует из теоремы 3 , найдется такая инфинитезимальная производящая функция $H(z, t)$ полугруппы $\mathfrak{L}[0,1]$ на промежутке $[0, T], T=\ln \beta$, что $f(z)=$ $w_{T, 0}^{H}(z)$. При этом в силу (4) должно выполняться равенство

$$
T=\int_{0}^{T} H(1, t) d t
$$


из которого следует, что $H(1, t)=1$ для почти всех $t \in[0, T]$. Для выделенной ветви логарифма имеем

$$
\frac{d}{d t} \ln \left\{\left(w_{t, 0}^{H}\right)^{\prime}(z)\right\}=\frac{1}{\left(w_{t, 0}^{H}\right)^{\prime}(z)} \frac{d}{d t}\left(w_{t, 0}^{H}\right)^{\prime}(z) .
$$

Меняя порядок дифференцирования, с учетом вида эволюционного уравнения (3) получаем

$$
\begin{aligned}
\frac{d}{d t}\left(w_{t, 0}^{H}\right)^{\prime}(z)=- & \left(w_{t, 0}^{H}\right)^{\prime}(z)\left[\left(1-w_{t, 0}^{H}(z)\right) H\left(w_{t, 0}^{H}(z), t\right)\right. \\
& \left.-w_{t, 0}^{H}(z) H\left(w_{t, 0}^{H}(z), t\right)+w_{t, 0}^{H}(z)\left(1-w_{t, 0}^{H}(z)\right) H^{\prime}\left(w_{t, 0}^{H}(z), t\right)\right] .
\end{aligned}
$$

Следовательно,

$$
\begin{aligned}
& \frac{d}{d t} \ln \left\{\left(w_{t, 0}^{H}\right)^{\prime}(z)\right\} \\
& \quad=-\left[\left(1-2 w_{t, 0}^{H}(z)\right) H\left(w_{t, 0}^{H}(z), t\right)+w_{t, 0}^{H}(z)\left(1-w_{t, 0}^{H}(z)\right) H^{\prime}\left(w_{t, 0}^{H}(z), t\right)\right] .
\end{aligned}
$$

Полагая в этом равенстве $z=0$, приходим к соотношению

$$
\frac{d}{d t} \ln \left\{\left(w_{t, 0}^{H}\right)^{\prime}(0)\right\}=-H(0, t)
$$

Поскольку $w_{0,0}^{H}(z) \equiv z$ и $w_{T, 0}^{H}(z)=f(z)$, то, интегрируя полученное соотношение по $t$ на промежутке $[0, T]$, получаем

$$
\ln f^{\prime}(0)=-\int_{0}^{T} H(0, t) d t
$$

Далее, условие $H(\cdot, t) \in \mathscr{Q}$ означает, что

$$
H(z, t)=\int_{\mathbb{T}} \frac{1-\varkappa}{1-\varkappa z} d \mu_{t}(\varkappa)
$$

где $\mu_{t}$ - вероятностная мера на $\mathbb{T}$. При этом $\mu_{t}(\mathbb{T} \backslash\{1\})=1$ в силу условия $H(1, t)=1$. Из равенства (13) следует теперь, что

$$
\ln f^{\prime}(0)=-\int_{0}^{T}\left(\int_{\mathbb{T}}(1-\varkappa) d \mu_{t}(\varkappa)\right) d t=-T+\int_{0}^{T}\left(\int_{\mathbb{T}} \varkappa d \mu_{t}(\varkappa)\right) d t .
$$

Отсюда получаем

$$
\ln f^{\prime}(0)=T(\omega-1),
$$

где $|\omega| \leqslant 1$ и $\omega \neq 1$. Это означает, что $\zeta=\ln f^{\prime}(0)$ и $\alpha=\ln f^{\prime}(1)=T$ удовлетворяют неравенству $|\zeta+\alpha| \leqslant \alpha$.

Допустим теперь, что выполняется равенство $|\zeta+\alpha|=\alpha$. Тогда $\varkappa_{0}=$ $(\zeta+\alpha) / \alpha$ принадлежит единичной окружности $\mathbb{T}$ и $\varkappa_{0} \neq 1$. Из предыдущего видно, что это возможно лишь в случае, когда для почти всех $t \in[0, T]$ 
(здесь $T=\alpha$ ) меры $\mu_{t}$ сосредоточены в точке $\varkappa_{0}$, т.е. $\mu_{t}\left(\left\{\varkappa_{0}\right\}\right)=1$. Другими словами, инфинитезимальная образующая в представлении $f(z)=w_{T, 0}^{H}(z)$ должна иметь вид

$$
H(z, t)=\frac{1-\varkappa_{0}}{1-\varkappa_{0} z}
$$

для почти всех $t \in[0, T]$. Из результатов $\S 6$ следует, что в этом случае

$$
f(z)=w_{T, 0}^{H}(z)=\phi_{\theta}^{\tau}(z)
$$

где $\theta \in(-\pi / 2, \pi / 2)$ определяется из равенства $e^{i 2 \theta}=-\varkappa_{0}$, а $\tau=2 T \cos \theta=|\zeta|$.

Теорема 4 доказана.

Пусть теперь $\mathfrak{L}_{1}(\beta), \beta>0$, - класс голоморфных и однолистных отображений $f$ единичного круга $\mathbb{D}$ в себя, которые оставляют неподвижной точку $z=1$ и имеют в ней угловую производную $f^{\prime}(1)=\beta$. Если $z_{0}-$ фиксированная точка в $\mathbb{D}$, то в силу теоремы Жюлиа-Каратеодори ее образ $w_{0}=f\left(z_{0}\right)$ при отображении посредством функции $f$ из $\mathfrak{L}_{1}(\beta)$ должен содержаться в орицикле, определяемом неравенством

$$
\frac{\left|1-w_{0}\right|^{2}}{1-\left|w_{0}\right|^{2}} \leqslant \beta \frac{\left|1-z_{0}\right|^{2}}{1-\left|z_{0}\right|^{2}}
$$

При этом равенство возможно лишь в случае, когда $f$ является дробно-линейным преобразованием единичного круга на себя.

Фиксируем пару точек $z_{0}, w_{0}$ в единичном круге $\mathbb{D}$ так, чтобы выполнялось неравенство

$$
\frac{\left|1-w_{0}\right|^{2}\left(1-\left|z_{0}\right|^{2}\right)}{\left(1-\left|w_{0}\right|^{2}\right)\left|1-z_{0}\right|^{2}}<\beta,
$$

и рассмотрим задачу об описании множества

$$
\mathscr{D}_{\beta}\left(z_{0}, w_{0}\right)=\left\{\zeta=f^{\prime}\left(z_{0}\right): f \in \mathfrak{L}_{1}(\beta), f\left(z_{0}\right)=w_{0}\right\} .
$$

Теорема 8. Пусть $\beta>0$ и пара точек $z_{0}, w_{0} \in \mathbb{D}$ удовлетворяют условию (14). Тогда имеет место теоретико-множественное равенство

$$
\mathscr{D}_{\beta}\left(z_{0}, w_{0}\right)=\left\{\zeta=\varkappa R e^{\omega}:|\omega| \leqslant \ln \frac{\beta}{\gamma}, \omega \neq \ln \frac{\beta}{\gamma}\right\},
$$

¿əe

$$
\varkappa=\frac{\left(1-\bar{z}_{0}\right)\left(1-w_{0}\right)}{\left(1-z_{0}\right)\left(1-\bar{w}_{0}\right)}, \quad R=\frac{1}{\beta} \frac{\left|1-w_{0}\right|^{2}}{\left|1-z_{0}\right|^{2}}, \quad \gamma=\frac{\left|1-w_{0}\right|^{2}\left(1-\left|z_{0}\right|^{2}\right)}{\left(1-\left|w_{0}\right|^{2}\right)\left|1-z_{0}\right|^{2}} .
$$

ДоказАтельство. Допустим вначале, что $\beta>0$ и пара точек $z_{0}, w_{0}$ из $\mathbb{D}$ удовлетворяет условию $(14)$, а функция $f$ принадлежит классу $\mathfrak{L}_{1}(\beta)$ и $f\left(z_{0}\right)=w_{0}$. Тогда функция $\varphi=S \circ f \circ L^{-1}$, где

$$
L(z)=\frac{1-\bar{z}_{0}}{1-z_{0}} \frac{z-z_{0}}{1-\bar{z}_{0} z}, \quad S(w)=\frac{1-\bar{w}_{0}}{1-w_{0}} \frac{w-w_{0}}{1-\bar{w}_{0} w},
$$


принадлежит классу $\mathfrak{L}[0,1]$. При этом

$$
\varphi^{\prime}(1)=\frac{\beta}{\gamma}, \quad f^{\prime}\left(z_{0}\right)=\varkappa \frac{1-\left|w_{0}\right|^{2}}{1-\left|z_{0}\right|^{2}} \varphi^{\prime}(0),
$$

где $\varkappa$ и $\gamma$ определены, как и в формулировке теоремы. В силу теоремы 4 значение $\ln \varphi^{\prime}(0)$ можно представить в виде

$$
\ln \varphi^{\prime}(0)=\omega-\ln \frac{\beta}{\gamma}, \quad|\omega| \leqslant \ln \frac{\beta}{\gamma}, \quad \omega \neq \ln \frac{\beta}{\gamma} .
$$

Следовательно,

$$
f^{\prime}\left(z_{0}\right)=\varkappa \frac{\gamma}{\beta} \frac{1-\left|w_{0}\right|^{2}}{1-\left|z_{0}\right|^{2}} e^{\omega}=\varkappa R e^{\omega} .
$$

Обратно, пусть $\zeta=\varkappa R e^{\omega},|\omega| \leqslant \ln (\beta / \gamma), \omega \neq \ln (\beta / \gamma)$, где $\varkappa, R$ и $\gamma$ определены, как в формулировке теоремы. Поскольку $\beta>\gamma$, то $\alpha=\ln (\beta / \gamma)>0$, и точка $(\alpha, \omega-\alpha)$ принадлежит множеству $\mathscr{D}$. Следовательно, найдется функция $\varphi$ из класса $\mathfrak{L}[0,1]$, для которой будут выполнены равенства

$$
\ln \varphi^{\prime}(1)=\alpha=\ln \frac{\beta}{\gamma}, \quad \ln \varphi^{\prime}(0)=\omega-\ln \frac{\beta}{\gamma} .
$$

Но тогда функция $f=S^{-1} \circ \varphi \circ L$, где дробно-линейные преобразования $S$ и $L$ определены выше, будет однолистно отображать единичный круг в себя. При этом $f$ оставляет неподвижной точку $z=1$ и имеет в ней угловую производную $f^{\prime}(1)=\beta$. Кроме того, $f\left(z_{0}\right)=w_{0}$ и

$$
f^{\prime}\left(z_{0}\right)=\varkappa \frac{1-\left|w_{0}\right|^{2}}{1-\left|z_{0}\right|^{2}} \varphi^{\prime}(0)=\varkappa \frac{\gamma}{\beta} \frac{1-\left|w_{0}\right|^{2}}{1-\left|z_{0}\right|^{2}} e^{\omega}=\varkappa R e^{\omega} .
$$

Теорема доказана.

Заметим также, что из теоремы 8 сразу следует неравенство

$$
\left|f^{\prime}(z)\right| \geqslant \frac{1}{\beta^{2}} \frac{\left(1-|z|^{2}\right)|1-f(z)|^{4}}{\left(1-|f(z)|^{2}\right)|1-z|^{4}},
$$

которое имеет место для любой функции $f$ из $\mathfrak{L}_{1}(\beta)$ и всех $z \in \mathbb{D}$. Этот результат другими методами был установлен ранее (см. [13]).

\section{Список литературы}

[1] K. Löwner, "Untersuchungen über schlichte konforme Abbildungen des Einheitskreises. I", Math. Ann., 89:1-2 (1923), 103-121.

[2] Г. М. Голузин, "О теоремах искажения в теории конформных отображений”, $M a$ тем. сб., 1(43):1 (1936), 127-135.

[3] L. de Branges, A proof of the Bieberbach conjecture, Preprint/LOMI E-5-84, Leningrad Branch of the V. A. Steklov Mathematical Institute, Leningrad, 1984, $21 \mathrm{pp}$.

[4] L. de Branges, "A proof of the Bieberbach conjecture", Acta Math., 154:1-2 (1985), $137-152$. 
[5] Г. М. Голузин, "Внутренние задачи теории однолистных функций", УМН, 1939, № $6,26-89$.

[6] П. П. Куфарев, "Об однопараметрических семействах аналитических функций”, Матем. сб., 13(55):1 (1943), 87-118.

[7] Ch. Pommerenke, "Über die Subordination analytischer Funktionen", J. Reine Angew. Math., 218 (1965), 159-173.

[8] В.Я. Гутлянский, "Параметрическое представление однолистных функций", Докл. АН СССР, 194 (1970), 750-753; англ. пер.: V. Ya. Gutlyanskii, "Parametric representation of univalent functions", Soviet Math. Dokl., 11 (1970), 1273-1276.

[9] В. В. Горяйнов, О. С. Кудрявцева, "Однопараметрические полугруппы аналитических функций, неподвижные точки и функция Кёнигса”, Матем. сб., 202:7 (2011), 43-74; англ. пер.: V. V. Goryainov, O. S. Kudryavtseva, "One-parameter semigroups of analytic functions, fixed points and the Koenigs function", Sb. Math., 202:7 (2011), 971-1000.

[10] В. В. Горяйнов, "Полугруппы аналитических функций в анализе и приложениях", УМН, 67:6(408) (2012), 5-52; англ. пер.: V.V. Goryainov, "Semigroups of analytic functions in analysis and applications", Russian Math. Surveys, 67:6 (2012), 975-1021.

[11] Э.А. Коддингтон, Н. Левинсон, Теория обыкновенных дифференииальных уравнений, ИЛ, М., 1958, 474 с.; пер. с англ.: Е. A. Coddington, N. Levinson, Theory of ordinary differential equations, McGraw-Hill Book Co., New York-Toronto-London, 1955, xii+429 pp.

[12] В.Н.Дубинин, "О граничных значениях производной Шварца регулярной функции”, Матем. сб., 202:5 (2011), 29-44; англ. пер.: V. N. Dubinin, "Boundary values of the Schwarzian derivative of a regular function", Sb. Math., 202:5 (2011), 649-663.

[13] В.Н.Дубинин, В. Ю. Ким, “Теоремы искажения для регулярных и ограниченных в круге функций", Аналитическая теория чисел и теория функиий. 22, Зап. научн. сем. ПОМИ, 350, ПОМИ, СПб., 2007, 26-39; англ. пер.: V. N. Dubinin, V. Yu. Kim, "Distortion theorems for functions regular and bounded in the disk", J. Math. Sci. (N. Y.), 150:3 (2008), 2018-2026.

[14] В.Н. Дубинин, "K неравенству Шварца на границе для регулярных в круге функций", Аналитическая теория чисел и теория функиий. 18, Зап. научн. сем. ПОМИ, 286, ПОМИ, СПб., 2002, 74-84; англ. пер.: V. N. Dubinin, "The Shwarz inequality on the boundary for functions regular in the disk", J. Math. Sci. (N. Y.), 122:6 (2004), 3623-3629.

[15] J. M. Anderson, A. Vasil'ev, "Lower Schwarz-Pick estimates and angular derivatives", Ann. Acad. Sci. Fenn. Math., 33:1 (2008), 101-110.

[16] Ch. Pommerenke, A. Vasil'ev, "Angular derivatives of bounded univalent functions and extremal partitions of the unit disk", Pacific J. Math., 206:2 (2002), 425-450.

[17] А.Ю. Солынин, "Граничное искажение и экстремальные задачи в некоторых классах однолистных функций", Аналитическая теория чисел и теория функuий. 11, Зап. научн. сем. ПОМИ, 204, Наука, СПб., 1993, 115-142; англ. пер.: A. Yu. Solynin, "The boundary distortion and extremal problems in certain classes of univalent functions", J. Math. Sci., 79:5 (1996), 1341-1358.

[18] Z. Nehari, "Some inequalities in the theory of functions", Trans. Amer. Math. Soc., 75:2 (1953), 256-286.

[19] A. Vasil'ev, Moduli of families of curves for conformal and quasiconformal mappings, Lecture Notes in Math., 1788, Springer-Verlag, Berlin, 2002, x+211 pp.

[20] L. V. Ahlfors, Conformal invariants: topics in geometric function theory, McGraw-Hill Series in Higher Mathematics, McGraw-Hill Book Co., New York-Düsseldorf-Johannesburg, 1973, ix+157 pp. 
[21] Ch. Pommerenke, Boundary behaviour of conformal maps, Grundlehren Math. Wiss., 299, Springer-Verlag, Berlin, 1992, x+300 pp.

[22] В.В. Горяйнов, “Эволюционные семейства конформных отображений с неподвижными точками", Збірник праць Ін-ту математики НАН України, 10:4-5 (2013), 424-431.

[23] В.В. Горяйнов, "О сходимости однопараметрических семейств аналитических функций”, Вопросъ метрической теории отображений и ее применение (Донецк, 1976), Наукова думка, Киев, 1978, 13-24.

[24] В. В. Горяйнов, "К параметрическому представлению однолистных функций", Докл. АН ССCP, 245:5 (1979), 1038-1041; англ. пер.: V. V. Goryainov, "On parametric representation of univalent functions", Soviet Math. Dokl., 20:2 (1979), $378-381$.

[25] Г. М. Голузин, Геометрическая теория функиий комплексного переменного, 2-е изд., Наука, М., 1966, 628 с.; англ. пер.: G. M. Goluzin, Geometric theory of functions of a complex variable, Transl. Math. Monogr., 26, Amer. Math. Soc., Providence, R.I., 1969, vi+676 pp.

[26] P. L. Duren, Univalent functions, Grundlehren Math. Wiss., 259, Springer-Verlag, New York, 1983, xiv+382 pp.

[27] В.В. Горяйнов, "Полугруппы конформных отображений", матем. сб., 129(171):4 (1986), 451-472; англ. пер.: V.V. Goryainov, "Semigroups of conformal mappings", Math. USSR-Sb., 57:2 (1987), 463-483.

\section{Виктор Владимирович Горяйнов (Viktor V. Goryainov)}

Московский физико-технический институт

(государственный университет),

г. Долгопрудный Московской обл.;

Волжский гуманитарный институт (филиал)

Волгоградского государственного университета

E-mail: goryainov_vv@hotmail.com 